\title{
Phase space quantization, noncommutativity and the gravitational field
}

\author{
Athanasios Chatzistavrakidis 1 \\ Institut für Theoretische Physik, Leibniz Universität Hannover, \\ Appelstraße 2, 30167 Hannover, Germany
}

\begin{abstract}
In this paper we study the structure of the phase space in noncommutative geometry in the presence of a nontrivial frame. Our basic assumptions are that the underlying space is a symplectic and parallelizable manifold. Furthermore, we assume the validity of the Leibniz rule and the Jacobi identities. We consider noncommutative spaces due to the quantization of the symplectic structure and determine the momentum operators that guarantee a set of canonical commutation relations, appropriately extended to include the nontrivial frame. We stress the important role of left vs. right acting operators and of symplectic duality. This enables us to write down the form of the full phase space algebra on these noncommutative spaces, both in the noncompact and in the compact case. We test our results against the class of $4 \mathrm{D}$ and $6 \mathrm{D}$ symplectic nilmanifolds, thus presenting a large set of nontrivial examples that realize the general formalism.
\end{abstract}

\footnotetext{
${ }^{1}$ thanasis@itp.uni-hannover.de
} 


\section{Contents}

1 Introduction $\quad$ 2

2 Quantum mechanics and noncommutativity 6

2.1 Phase space of the noncommutative plane and torus . . . . . . . . . . 6

2.2 Left vs. right action and symplectic duality . . . . . . . . . . . 9 9

3 Quantized phase spaces and curvature 10

3.1 Introduction of curvature . . . . . . . . . . . . . . . . . . 10

3.2 Momentum operators in the presence of a nontrivial frame . . . . . . . 12

4 Quantized phase space of symplectic nilmanifolds

4.1 Step classification of $4 \mathrm{D}$ and $6 \mathrm{D}$ nilmanifolds . . . . . . . . . . 16

4.2 Determining the phase space algebra . . . . . . . . . . . . . 19

4.3 Remarks on the compact case . . . . . . . . . . . . . . . 21

5 Benchmark examples of quantized phase spaces 22

5.1 Dimension $4 \ldots \ldots \ldots \ldots \ldots \ldots \ldots \ldots$. . . . . . . . . . . . . . . . . . . . . .

5.2 Dimension $6 \ldots \ldots \ldots \ldots \ldots \ldots$

6 Discussion 28

A Additional data for symplectic nilmanifolds 


\section{Introduction}

The most challenging conceptual problem of modern theoretical physics is the lack of a complete understanding of the physics of phenomena related to the fundamental constants $G_{N}$ and $\hbar$. These are quantum gravitational phenomena that become important near the Planck scale, defined by the mass scale $m_{P}=\sqrt{\frac{\hbar c}{G_{N}}}$, or by the length scale $l_{P}=\sqrt{\frac{\hbar G_{N}}{c^{3}}}$.

Quantum field theory describes physical processes where both c and $\hbar$ are important. In this sense, it is a unifying framework for special relativity and quantum mechanics. Its success is unquestionable, since it successfully incorporates three of the four fundamental interactions, the electromagnetic, weak and strong ones, in a particular unifying theory, the standard model. The latter has achieved unprecedented agreement with experimental data and a unique corroboration of its merit as a valid theory, at least up to energy scales of $1 \mathrm{TeV}$.

Incorporating gravitational interactions in a unifying scheme with the rest of the forces is a notoriously difficult problem. String theory is at present the only framework where this is possible in a mathematically consistent way. The fundamental degrees of freedom in string theory are one-dimensional objects that do not propagate on a predetermined spacetime continuum, but instead they determine the geometry of spacetime. Indeed, the concept of spacetime and its dynamics is a derived or emergent concept that arises from the quantization of the two-dimensional non-linear sigma model that models the propagation of a string world sheet.

The fact that strings are extended objects means that they cannot be associated to points in spacetime. This indicates that string geometry should have no points, unlike classical differential geometry. Pointless geometries are best accommodated in the mathematical framework of noncommutative geometry [1-3]. In such geometries, the spacetime coordinates become noncommuting operators and therefore a single point cannot by definition be resolved in any thought experiment, much like points in the phase space of quantum mechanics. This close relation between the geometry probed by a string and noncommutative spacetime geometries has been confirmed through the many connections that were established between string theory and noncommutative geometry [4,5] (see also Ref. 6] for a review of more recent progress and the related literature). In this sense, these two frameworks are close collaborators regarding questions of quantum gravitational phenomena.

String theory and noncommutative geometry introduce their respective scales, namely the string length $l_{s}$ in the first case and the length scale $l_{\mathrm{NC}}$ where the classical description of spacetime is lost in the second. Equivalently one can think in terms of the string slope parameter $\alpha^{\prime}$, which is equal to the square of the string length, or a noncommutativity scale $l_{\mathrm{NC}}^{2}$ that appears in the commutator of coordinate operators in a noncommutative algebra. The above arguments suggest that there should exist a relation $\alpha^{\prime} \sim l_{\mathrm{NC}}^{2}$ among the two. However, the generally accepted statement that at extremely small distances the classical notion of spacetime breaks down and it has to be replaced by some notion of quantum or fuzzy spacetime can be supported with arguments that are independent of string theory too [1,7].

The physical motivation for the present work is to derive some lessons on the interplay between quantum mechanics and gravity through noncommutative geometry. In order to 
do so, one has to understand how the scales $G_{N}$ and $\hbar$ come together in a noncommutative algebra. This can be understood in the context of a noncommutative phase space, whose algebraic structure is in general

$$
\begin{aligned}
{\left[\hat{x}^{a}, \hat{x}^{b}\right] } & =i l_{\mathrm{NC}}^{2} \theta^{a b}\left(\hat{x}^{c}\right) \\
{\left[\hat{x}^{a}, \hat{p}_{i}\right] } & =i \hbar e^{a}{ }_{i}\left(\hat{x}^{c}\right)=i \hbar \delta_{i}^{a}+i m_{\mathrm{NC}} f_{i b}^{a} \hat{x}^{b}+\mathcal{O}\left(\hat{x}^{2}\right) \\
{\left[\hat{p}_{i}, \hat{p}_{j}\right] } & =i \frac{\hbar^{2}}{l_{\mathrm{NC}}^{2}} F_{i j}
\end{aligned}
$$

where all the undefined quantities will be explained in detail below. What we point out here are the scales that appear in the algebra2 2 . As already discussed, the length scale $l_{\mathrm{NC}}$ should be related to a small fundamental length, for example the Planck length $l_{\mathrm{NC}}^{2} \sim G_{N} \hbar$ (or the string length, if one wishes to relate the two scales). Moreover, we denoted as $m_{\mathrm{NC}}$ the combination $\hbar / l_{\mathrm{NC}}$ (recall that $c=1$ ) and this should be related to the Planck mass, $m_{\mathrm{NC}}^{2} \sim \hbar / G_{N}$. It will become clear in the following that turning off the gravitational field the commutation relation between positions and momenta becomes the canonical commutation relation of quantum mechanics, and the momenta commute, as long as there are no magnetic sources in the problem. Thus turning off gravity we get noncommutative quantum mechanics and furthermore standard quantum mechanics in the limit $l_{\mathrm{NC}} \rightarrow 0$.

The reason that we consider the full phase space instead of just the commutator of coordinates should be clear by the fact that quantum mechanics appears as a limiting case. Indeed, quantum mechanics teaches us that the phase space is an essential concept in the understanding of the underlying physics, a fact that is sometimes overlooked in applications of noncommutative geometry in high-energy physics. It is reasonable to expect that phase space plays an equally important role in quantum gravity 3 . It should be mentioned that this was already emphasized long ago by Madore [1, who examined the role and properties of momenta in noncommutative geometry within the noncommutative frame formalism. More recent developments in this framework include Refs. [8 10]. The importance of noncommutative phase space in physical problems, such as quantum particles in strong magnetic fields, was also emphasized in Refs. [11,15].

In this paper we are interested in examining the algebraic properties of the phase space when it is quantized in the presence of a nontrivial frame. The main physical reason to do this is that the frame is associated to the gravitational field. Therefore we expect to get some first lessons for physical problems that involve the behavior of quantum particles in the presence of gravity. These are situations that generalize the cases studied in Refs. [11-18, relevant for physical problems such as quantum particles moving in electromagnetic fields or the quantum Hall effect. On the other hand, although the noncommutativity of phase space in quantum mechanics was based on experimental facts, there is no experimental result yet that points to the phase space we describe here. However there are good conceptual reasons to consider it, as described above, and moreover one could hope for some basic experimental support of the general framework by experiments such as the Fermilab Holometer [19, 20], which is designed to test proposals associated to the quantization of spacetime.

\footnotetext{
${ }^{2}$ We set $c=1$, since we are not interested in the corresponding physics here.

${ }^{3}$ This statement can be made more precise once a dynamical theory for quantum fields in phase space that incorporates the gravitational field is established. Although we do not directly address this problem in the present paper, we discuss a possible way to achieve this goal in the discussion section.
} 
In order to carry out the above task, one basic assumption we make is that the spaces we investigate admit a symplectic structure. It is well-known that symplectic manifolds have tractable quantization properties, either via deformation quantization [21] or Weyl quantization [22]. Moreover, we assume that the space is parallelizable so that a globally well-defined frame exists on it. Nontrivial symplectic, parallelizable and curved manifolds exist and we are going to provide a class of examples, the symplectic nilmanifolds.

At this point it is useful to recall that in classical mechanics in d-dimensional flat space, the Hamiltonian formalism includes a set of coordinates $x^{a}, a=1, \ldots, \mathrm{d}$ and momenta $p_{a}$, building up a $2 \mathrm{~d}$-dimensional phase space in $\mathrm{d}$ dimensions. This phase space has the structure of a symplectic manifold with symplectic structure,

$$
\omega=\delta_{a}^{b} \mathrm{~d} x^{a} \wedge \mathrm{d} p_{b},
$$

where summation is implied. $x^{a}$ and $p_{a}$ are the canonical coordinates with differentials (1forms) $\mathrm{d} x^{a}$ and $\mathrm{d} p_{a}$. The corresponding dual derivations are $\partial_{x^{a}}=\partial / \partial x^{a}$ and $\partial_{p_{a}}=\partial / \partial p_{a}$. The symplectic structure defines a Poisson bracket, given as

$$
\{f, g\}=\delta_{a}^{b}\left(\partial_{x^{b}} f \partial_{p_{a}} g-\partial_{p_{a}} f \partial_{x^{b}} g\right) \text {. }
$$

In particular,

$$
\left\{x^{a}, p_{b}\right\}=\delta_{b}^{a}
$$

On the other hand, in quantum mechanics, where $x^{a}$ and $p_{a}$ become hermitian operators, the structure of $\mathrm{d} x^{a}, \mathrm{~d} p_{a}, \partial_{x^{a}}$ and $\partial_{p_{a}}$ exhibits a degree of redundancy in the following sense. Since the wave function $\Psi(x)$ in the coordinate representation is a function of the positions only and not of both positions and momenta $\Psi(x, p)$, the quantum physics in the coordinate representation does not involve $\partial_{p_{a}}$ at all. Indeed, the canonical commutation relation $(\mathrm{CCR})$

$$
\left[\hat{x}^{a}, \hat{p}_{b}\right]=i \hbar \delta_{b}^{a}
$$

which can be thought of as the quantization of the Poisson bracket 4 , is represented on the Hilbert space by the operators

$$
\hat{x}^{a} \Psi=x^{a} \Psi, \quad \hat{p}_{a} \Psi=-i \hbar \delta_{a}^{b} \partial_{x^{b}} \Psi
$$

in accord with the Stone-von Neumann theorem. In the dual picture of the momentum representation, where the wave function depends on the momenta $\Psi(p)$, the operators are represented as

$$
\hat{x}^{a} \Psi=i \hbar \delta_{b}^{a} \partial_{p_{b}} \Psi, \quad \hat{p}_{a} \Psi=p_{a} \Psi .
$$

Of course there is a continuum of intermediate mixed pictures but these are not particularly useful. In any case, a simultaneous consideration of $\partial_{x^{a}}$ and $\partial_{p_{a}}$ is unnecessary. From a different point of view, employing the position representation, there is a commutative algebra of operators $\hat{x}^{a}$ and the momentum operators are included as outer derivations

\footnotetext{
${ }^{4}$ In the sense of Dirac, where the replacement $\{\cdot, \cdot\} \rightarrow \frac{1}{i \hbar}[\cdot, \cdot]$ accounts for quantization. This simple relation should be treated cautiously, since it is not a sufficient and complete rule. For more details see for example the lecture notes 23 .
} 
in the algebra and thus they do not belong to the algebra which the position operators generate.

On the contrary to the latter statement, in noncommutative geometry the momenta do not necessarily correspond to outer derivations and they can as well be elements of the noncommutative algebra $\mathcal{A}$ generated by the position operators; namely they can also be inner. Thus, the starting point is the noncommutative but associative algebra $\mathcal{A}$ of coordinate operators and the momenta can be formally expressed in terms of the coordinate operators, $\hat{p}_{a}=\hat{p}_{a}\left(\hat{x}^{b}\right)$. This leads to a picture where the full phase space is associated to a noncommutative algebra where not only coordinates but also momenta do not necessarily commute among themselves. In the simplest case of noncommutative quantum mechanics in the absence of curvature, the CCRs are retained and they are supplemented by commutation relations among coordinates and among momenta separately. In the absence of sources and gravity, the momenta commute. However, this is not anymore true where sources are included or the gravitational field is present.

In the present work we are interested in the case of the gravitational field, associated with a frame $e^{i}{ }_{a}$. As already stated, we assume that the space is parallelizable and it admits a symplectic structure and that the symplectic 2-form $\omega$, as well as the corresponding symplectic 2 -vector $\theta$, is constant in the basis of the globally well-defined frame. noncommutativity is introduced in the commutator of the position operators, setting it equal to the components of the symplectic structure in the curved basis,

$$
\left[\hat{x}^{a}, \hat{x}^{b}\right]=i \theta^{a b}\left(\hat{x}^{c}\right)
$$

where from now on we set $l_{\mathrm{NC}}=1$. In this basis the parameters are not necessarily constant. However, we will see that there exists an interesting class of noncommutative spaces with curvature where they are constant in a chosen coordinate system. In the presence of a nontrivial frame $e^{i}{ }_{a}$ with inverse $e_{i}^{a}$, the appropriate commutation relations between momenta and coordinates are augmented to

$$
\left[\hat{x}^{a}, \hat{p}_{i}\right]=i \hbar e^{a}{ }_{i}\left(\hat{x}^{c}\right)
$$

where on the right hand side we encounter the noncommutative frame which is related to the gravitational field [9]. In the following we will find the expression for the momenta and show how the full phase space algebra is determined. This algebra is required to satisfy the Jacobi identities. Going one step further, we depart from the noncompact case and study the phase space algebra when a periodicity condition that compactifies the space is imposed. This is analogous to the condition that compactifies a d-plane to a d-torus. In such cases, the operators $\hat{x}^{a}$ turn out to be unphysical and the correct position operators are obtained with exponentiation of $\hat{x}^{a}$. Moreover, we test our results both in the noncompact and compact cases in a class of explicit examples, the symplectic nilmanifolds in four and six dimensions.

\footnotetext{
${ }^{5}$ Throughout this paper, $i, j, \ldots$ are flat (tangent space) indices, while $a, b, \ldots$ are curved (world) indices.
} 


\section{Quantum mechanics and noncommutativity}

\subsection{Phase space of the noncommutative plane and torus}

In standard quantum mechanics, phase space is noncommutative. This is just the statement that a nontrivial commutation relation between positions and momenta exists, the CCR, which is the basis of the uncertainty principle. However, both the position space and the momentum space are commutative. The full phase space algebra is simply

$$
\left[\hat{x}^{i}, \hat{x}^{j}\right]=0, \quad\left[\hat{x}^{i}, \hat{p}_{j}\right]=i \hbar \delta_{j}^{i}, \quad\left[\hat{p}_{i}, \hat{p}_{j}\right]=0
$$

where we use flat indices for both positions and momenta6. As discussed in the introduction, this algebra of operators can be represented in the position representation, where the eigenvalues of the Hermitian operators $\hat{x}^{i}$ are ordinary real numbers $x^{i} \in \mathbb{R}$ and the momentum operators are translations, namely partial derivatives with respect to $x^{i}$, or in the dual momentum representation where the roles are exchanged. As we already stressed above, the momenta are introduced as outer derivations in the algebra of position operators. In standard quantum mechanics the momentum operators cannot be inner derivations of the algebra.

On the other hand, one can consider the quantum mechanics of particles on a noncommutative space, as for example in Refs. [11 15, 24]. The simplest possibility is a noncommutative plane in d dimensions or a noncommutative d-torus. Although these cases are well known, let us review the main steps and results in order to warm up for the more general cases that we will present in the rest of this paper.

Let us first consider the noncompact case of a noncommutative d-plane of even dimension. This is specified by a set of coordinate operators which satisfy a commutation relation of the form $\left[\hat{x}^{i}, \hat{x}^{j}\right]=i \theta^{i j}$. Here, $\theta^{i j}$ is the set of constant noncommutativity parameters. They can be identified with the components of a symplectic structure on the corresponding classical manifold, namely with a constant symplectic 2-vector in the globally well-defined basis. The quantum mechanics of particles on this space is associated with the noncommutative phase space algebra

$$
\left[\hat{x}^{i}, \hat{x}^{j}\right]=i \theta^{i j}, \quad\left[\hat{x}^{i}, \hat{p}_{j}\right]=i \hbar \delta_{j}^{i}, \quad\left[\hat{p}_{i}, \hat{p}_{j}\right]=0
$$

which extends the standard algebra $(2.1)]^{7}$. The prime question to address concerns the realization of this algebra, which cannot be the same as in standard quantum mechanics for obvious reasons. One way to think about this problem is to associate $\hat{x}^{i}$ to a set of Hermitian matrices from a matrix algebra $\mathcal{A}$ (in particular the Heisenberg algebra in $\mathrm{d}$ dimensions) and the momenta to inner derivations of the algebra $\mathcal{A}$, similarly to Ref. [25]. Although representing momenta with inner derivations was impossible in standard quantum

\footnotetext{
${ }^{6}$ In the present case the classical globally well defined 1-forms are simply $e^{i}=\delta_{a}^{i} \mathrm{~d} x^{a}=\mathrm{d}\left(\delta_{a}^{i} x^{a}\right)$ and therefore we can introduce flat coordinates $x^{i}=\delta_{a}^{i} x^{a}$ and the corresponding quantum operators. Similarly, the dual vector fields are just $\theta_{i}=\delta_{i}^{a} \partial_{a}$ and we can define $\hat{p}_{i}=\delta_{i}^{a} \hat{p}_{a}$. Thus we can work fully in flat indices instead of cluttering with the tensors $\delta_{i}^{a}$ and $\delta_{a}^{i}$.

${ }^{7} \mathrm{We}$ are interested in the case where no magnetic sources are present. Otherwise the commutator of the momenta is also nonvanishing and proportional to the magnetic field [13].
} 
mechanics, it is working perfectly in the noncommutative case. Inner derivations in matrix algebras act with the adjoint action and therefore we consider the following Ansatz for the momenta,

$$
\hat{p}_{i}=c_{i j} \operatorname{ad} \hat{x}^{j}=c_{i j}\left[\hat{x}^{j}, \cdot\right],
$$

where $\cdot$ is a placeholder for arbitrary elements of the algebra $\mathcal{A}$ and $c_{i j}$ are constants to be determined. First we consider the CCRs with the position operators. Acting on an arbitrary function $f \in \mathcal{A}$ they imply

$$
\begin{aligned}
i \hbar \delta_{i}^{j} f & =\left[\hat{x}^{j}, \hat{p}_{i}\right] f=\hat{x}^{j} c_{i k}\left[\hat{x}^{k}, f\right]-c_{i k}\left[\hat{x}^{k}, \hat{x}^{j} f\right]= \\
& =c_{i k} \hat{x}^{j}\left[\hat{x}^{k}, f\right]-c_{i k} \hat{x}^{j}\left[\hat{x}^{k}, f\right]-c_{i k}\left[\hat{x}^{k}, \hat{x}^{j}\right] f= \\
& =-i c_{i k} \theta^{k j} f
\end{aligned}
$$

where we used the fact that $c_{i j}$ are constant and we applied the Leibniz rule. Then we obtain

$$
c_{i j}=\hbar \delta_{i}^{k} \omega_{k j}=\hbar \omega_{i j},
$$

where $\omega_{i j}$ are the constant components of the non-degenerate symplectic 2-form which satisfies the relation

$$
\theta^{i j} \omega_{j k}=-\delta_{k}^{i}
$$

with the symplectic 2 -vector components. Therefore the momenta are given as

$$
\hat{p}_{i}=\hbar \omega_{i j}\left[\hat{x}^{j}, \cdot\right]
$$

Their trivial commutation relation remains to be examined. We compute

$$
\begin{aligned}
0 & =\left[\hat{p}_{i}, \hat{p}_{j}\right] f=\hbar \omega_{i k}\left[\hat{x}^{k}, \hbar \omega_{j l}\left[\hat{x}^{l}, f\right]\right]-\hbar \omega_{j l}\left[\hat{x}^{l}, \hbar \omega_{i k}\left[\hat{x}^{k}, f\right]\right]= \\
& =\hbar^{2} \omega_{i k} \omega_{j l}\left(\left[\hat{x}^{k},\left[\hat{x}^{l}, f\right]\right]-\left[\hat{x}^{l},\left[\hat{x}^{k}, f\right]\right]\right)= \\
& =\hbar^{2} \omega_{i k} \omega_{j l}\left[\left[\hat{x}^{k}, \hat{x}^{l}\right], f\right]=i \hbar^{2} \omega_{i k} \omega_{j l}\left[\theta^{k l}, f\right]
\end{aligned}
$$

which holds because of the constancy of $\theta^{k l}$. In this computation the only additional input that has to be used is the Jacobi identity in the algebra $\mathcal{A}$, which we assume is valid.

A different but equivalent point of view is to write the Ansatz for the momentum operators as

$$
\hat{p}_{i}=c_{i j}\left(\hat{x}^{j}+\hat{y}^{j}\right),
$$

with some appropriate operators $\hat{y}^{j}$. Under the assumption that

$$
\left[\hat{x}^{i}, \hat{y}^{j}\right]=0
$$

the commutation relation with $\hat{x}^{i}$ fixes $c_{i j}$ to be $\hbar \omega_{i j}$, as before. Then the vanishing commutator among the momenta gives

$$
0=\left[\hat{p}_{i}, \hat{p}_{j}\right]=\hbar^{2} \omega_{i k} \omega_{j l}\left(\left[\hat{x}^{k}, \hat{x}^{l}\right]+\left[\hat{y}^{k}, \hat{y}^{l}\right]\right) \quad \Rightarrow \quad\left[\hat{y}^{i}, \hat{y}^{j}\right]=-\left[\hat{x}^{i}, \hat{x}^{j}\right]
$$

This means that

$$
\left[\hat{y}^{i}, \hat{y}^{j}\right]=-i \theta^{i j}
$$


In other words, the momenta are realized with two mutually commuting copies of the algebra $\mathcal{A}$. Although this might seem puzzling, it is fully equivalent to the previous approach, as explained in Ref. [25]. The equivalence is established upon the identification

$$
\hat{y}^{i}=-\hat{x}_{R}^{i},
$$

where $\hat{x}_{R}^{i}$ denotes the generators of the $\mathcal{A}_{R}$ copy of the algebra $\mathcal{A}$ which act on states from the right, instead of acting from the left as implicitly assumed up to now. The right and left acting copies of $\mathcal{A}$ are mutually commuting indeed. Then the momenta become

$$
\hat{p}_{i}=\hbar \omega_{i j}\left(\hat{x}_{L}^{j}-\hat{x}_{R}^{j}\right),
$$

which is the same as the expression (2.7). It should be appreciated that whenever a commutator action is encountered in noncommutative theories, there are two copies of the algebra $\mathcal{A}$ involved, the left and the right acting ones. We will see in the following that this distinction between left and right acting operators becomes crucial in more involved cases than the planar one.

The above considerations provide an understanding of the phase space algebra of noncommutative quantum mechanics and its realization in the absence of sources. The next step is to consider the compact case, which corresponds to a noncommutative torus. We follow the analysis of Ref. [13] in order to illustrate this case. The starting point of the analysis is that the standard periodicity condition of a d-torus has to be imposed, i.e.

$$
\hat{x}^{i} \sim \hat{x}^{i}+2 \pi R^{j} \delta_{j}^{i}
$$

where $R_{i}$ are the $\mathrm{d}$ radii of the corresponding cycles, not necessarily equal for a rectangular torus. The central observation is that due to this condition, the operators $\hat{x}^{i}$ are not single valued and therefore they are unphysical, i.e. they do not correspond to observables. The physical operators of positions on the noncommutative torus are obtained by exponentiation as

$$
X^{i}=e^{i b_{j}^{i} \hat{x}^{j}}
$$

where $b_{j}^{i}$ are constants to be determined. This is achieved by demanding the unitary operators $X^{i}$ to be globally well defined, i.e. to be invariant under the shift (2.15). The condition for this to happen is

$$
i b_{j}^{i} 2 \pi R^{k} \delta_{k}^{j}=2 \pi i N \delta_{k}^{i}, \quad N \in \mathbb{N},
$$

which gives

$$
b_{j}^{i}=\frac{N}{R^{j}} \delta_{j}^{i} .
$$

This results in the well-defined operators

$$
X^{i}=e^{\frac{i \hat{x}^{i}}{R^{i}}},
$$

where we set $N=1$ for simplicity. Then the stage is set to write down the full phase space algebra for the noncommutative torus. The momenta are given as before and they are now outer derivations instead of inner, which was the noncompact case. The algebra is

$$
\begin{aligned}
X^{i} X^{j} & =e^{-\frac{i \theta^{i j}}{R^{i} R^{j}}} X^{j} X^{i}, \\
\hat{p}_{i} X^{j} & =X^{j}\left(\hat{p}_{i}+\frac{\hbar}{R^{j}} \delta_{i}^{j}\right), \\
{\left[\hat{p}_{i}, \hat{p}_{j}\right] } & =0,
\end{aligned}
$$


in agreement with the approach of Ref. [4] on noncommutative tori. Representations and quantum bundles over this algebra were discussed in detail in Refs. [13, 26]. Here we are interested in the generalization of the above phase space algebras in the presence of a nontrivial frame.

\subsection{Left vs. right action and symplectic duality}

Before introducing curvature, it is useful to discuss further the left and right realizations of the noncommutative algebra $\mathcal{A}$. To this end we return to curved indices and we consider the algebra $\mathcal{A}$, generated by $\hat{x}^{a}$. The latter satisfy the relation $\left[\hat{x}^{a}, \hat{x}^{b}\right]=i \theta^{a b}$, with the parameters $\theta^{a b}$ being in the curved basis and therefore not necessarily constant. We denote as $\hat{x}_{L}^{a}$ the left acting position operators and as $\hat{x}_{R}^{a}$ the right acting ones, and $\mathcal{A}_{L}$ and $\mathcal{A}_{R}$ denote the corresponding algebras. Clearly, $\left[\hat{x}_{L}^{a}, \hat{x}_{L}^{b}\right]=i \theta^{a b}$. It is also obvious that $\left[\hat{x}_{L}^{a}, \hat{x}_{R}^{b}\right]=0$ in full generality. On the other hand, for $f \in \mathcal{A}$ we find

$$
\left[\hat{x}_{R}^{a}, \hat{x}_{R}^{b}\right] f=\left(\hat{x}_{R}^{a} \hat{x}_{R}^{b}-\hat{x}_{R}^{b} \hat{x}_{R}^{a}\right) f=f\left(\hat{x}^{b} \hat{x}^{a}-\hat{x}^{a} \hat{x}^{b}\right)=f\left(-i \theta^{a b}\right)=-i \theta^{a b} f+i\left[\theta^{a b}, f\right] .
$$

In the flat case, the last term on the right-hand side is zero and then $\hat{x}_{R}^{a}$ form a copy of the algebra $\mathcal{A}$, as in Section 2.1. This is not generally true when the curved components of the symplectic structure are not constant. In order to be able to proceed further, two additional minimal assumptions are due. First, we demand that $f \in \mathcal{A}_{L}$, a very mild assumption which is made anyway in all similar approaches. Secondly, in order to obtain $\left[\theta^{a b}, f\right]=0$ with general $\theta^{a b}$, we assume that these components depend on the right acting operators $\hat{x}_{R}^{a}$ if they are not constant. Then due to the commutativity between left and right acting operators, we get

$$
\left[\hat{x}_{R}^{a}, \hat{x}_{R}^{b}\right] f=-i \theta^{a b} f .
$$

This means that the full set of relations is

$$
\left[\hat{x}_{L}^{a}, \hat{x}_{L}^{b}\right]=i \theta^{a b}, \quad\left[\hat{x}_{L}^{a}, \hat{x}_{R}^{b}\right]=0, \quad\left[\hat{x}_{R}^{a}, \hat{x}_{R}^{b}\right]=-i \theta^{a b} .
$$

The different sign of the left and right commutators makes manifest the symplectic duality among the two sets. The concept of symplectic dual is very simple. Given a symplectic manifold with symplectic structure $\omega$, its symplectic dual is again a symplectic manifold based on the same underlying manifold and an opposite symplectic structure $-\omega[23$. This symplectic duality is elegantly realized in the context that we examine here.

Let us now take a look at the momentum operators too. In the flat case, the CCR $\left[\hat{x}^{j}, \hat{p}_{i}\right]=$ $i \hbar \delta_{i}^{j}$ holds as it is for the left acting position operators. However, it is a straightforward calculation to show that it holds the same for the right acting ones too, i.e.

$$
\left[\hat{x}_{R}^{j}, \hat{p}_{i}\right]=i \hbar \delta_{i}^{j}
$$

This obviates the need for right acting momentum operators. This should be expected from the fact that these operators already involve both the left and right acting position operators. On the other hand, in the curved case we will show that although $\left[\hat{x}_{L}^{a}, \hat{p}_{i}\right]=i \hbar e^{a}{ }_{i}$, in the most interesting cases it holds that

$$
\left[\hat{x}_{R}^{a}, \hat{p}_{i}\right]=i \hbar e_{i}^{a}+\hbar \omega_{c b}\left[\hat{x}_{R}^{a}, e_{i}^{c}\right]\left(\hat{x}_{L}^{b}-\hat{x}_{R}^{b}\right)
$$


This shows that left and right operators are liberated and they play asymmetric roles in the formalism. Finally, it should be clear that had we focused on the momentum representation, the roles of momentum and position operators would have been fully exchanged, as in standard quantum mechanics. In this paper we work on the position representation.

\section{Quantized phase spaces and curvature}

\subsection{Introduction of curvature}

In the previous section we reviewed the phase spaces of standard quantum mechanics, noncommutative planar quantum mechanics and noncommutative toroidal quantum mechanics. In all cases the space is flat and there is no sign of the gravitational field. This is evident by the fact that only flat indices appear. Essentially, the flat tensor $\delta_{a}^{i}$ is implicitly present in all the formulas, through the relation $\hat{x}^{i}=\delta_{a}^{i} \hat{x}^{a}$ that holds in the flat case.

An elegant way to introduce the gravitational field is through the vielbein and the frame formalism, as for example in the treatment of gravity as a gauge theory (see e.g. the textbook [27] for a concise presentation). There one substitutes 8

$$
\delta^{i}{ }_{a} \rightarrow e^{i}{ }_{a}(x)
$$

This substitution indicates that the CCRs are augmented to a set of extended CCRs (EC$\mathrm{CRs})$

$$
\left[\hat{x}^{a}, \hat{p}_{i}\right]=i \hbar e_{i}^{a}\left(\hat{x}^{b}\right) .
$$

Such an approach was advocated and followed in Refs. 8-10] and many aspects of our approach are similar, although not identical. In Eq. (3.2), $e_{i}^{a}$ is the (inverse of the) noncommutative vielbein, obtained by the commutative one upon promoting the coordinates to operators, as in standard quantum mechanics. Note that in Section 2 we emphasized the role of left and right acting operators. Although we indicated the dependence of the frame as $\hat{x}^{b}$, it is not a priori clear which of the two sets of operators is to be taken. Our approach is to keep an open mind and let the consistency of the formalism decide. It will in fact turn out that the frame depends on the right acting set of operators $\hat{x}_{R}^{b}$.

Let us give a list of our assumptions, which we already mentioned in the introduction, with some additional details. First we assume that the classical manifold admits a symplectic structure. In other words it is endowed with a nondegenerate closed 2-form $\omega, \mathrm{d} \omega=0$, which is invertible. Second, we assume that the classical manifold is parallelizable and therefore it admits a set of globally well-defined 1-forms $e^{i}$ which serve as a basis of its cotangent bundle. In this basis the symplectic 2-form is assumed to have constant coefficients. In

\footnotetext{
${ }^{8} \mathrm{Up}$ to now we did not bother about the horizontal position of the indices, since only the flat tensor appeared where there is no difference when the inverse or the transpose is taken. From now on the position is important so let us explain how we denote these tensors. We do not use an explicit notation for the inverse or the transpose. It should be clear from the index structure. The inverse of $e^{i}{ }_{a}$ is $e^{a}{ }_{i}$ and the transpose is $e_{a}^{i}$. The inverse transpose is $e_{i}{ }^{a}$. Also we often refrain from explicitly writing the $x$-dependence of all these tensors.
} 
flat space the $e^{i}$ 's are just $\mathrm{d} x^{i}$, but this is no longer true for an arbitrary, possibly curved, manifold. In general, they are related to an explicit coordinate basis by

$$
e^{i}=e_{a}^{i}(x) \mathrm{d} x^{a} .
$$

In geometric terms $e_{a}^{i}(x)$ is the twist matrix which relates the two bases. It is invertible and its inverse is denoted as $e_{i}^{a}(x)$, as already stated; thus

$$
\mathrm{d} x^{a}=e_{i}^{a} e^{i} .
$$

In gravitational language, it defines the gravitational field, relating flat and curved indices. Its exterior derivative is a 2-form, which can be expanded in the basis of the cotangent bundle,

$$
\mathrm{d} e^{i}=-\frac{1}{2} f^{i}{ }_{j k} e^{j k}
$$

where from now on we use the notation $e^{i j}=e^{i} \wedge e^{j}$. These are essentially the MaurerCartan equations. Solving for the coefficients, we get

$$
f_{j k}^{i}=2 e_{[j}^{a} e_{k]}^{b} \partial_{b} e^{i}{ }_{a} .
$$

The symplectic 2-form can be expanded in the basis 2-forms too. It has the form

$$
\omega=\frac{1}{2} \omega_{i j} e^{i j}
$$

where $\omega_{i j}$ is independent of $x^{a}$. It is useful to present its components in the curved basis as well. We compute

$$
\omega=\frac{1}{2} \omega_{i j} e_{a}^{i} e_{b}^{j} \mathrm{~d} x^{a b} \quad \Rightarrow \quad \omega_{a b}=\omega_{i j} e_{[a}^{i} e_{b]}^{j} .
$$

The symplectic 2-vector has the form

$$
\theta=\frac{1}{2} \theta^{i j} \theta_{i} \wedge \theta_{j},
$$

where the $\theta_{i}$ 's are the dual vectors to the basis 1 -forms $e^{i}$. Its components are opposite to the components of the inverse of the symplectic 2 -form, namely

$$
\theta^{i j}=-\left(\omega^{-1}\right)^{i j} .
$$

Obviously, it holds that

$$
\theta^{i j} \omega_{j k}=-\delta_{k}^{i}
$$

and similarly for the curved indices.

Moreover, we assume the validity of the Leibniz rule and the Jacobi identities. The first means that for any three functions $f, g, h \in \mathcal{A}_{L}$

$$
[f, g h]=g[f, h]+[f, g] h .
$$

The Jacobi identities are

$$
\operatorname{Jac}(f, g, h):=[f,[g, h]]+[h,[f, g]]+[g,[h, f]]=0 .
$$


These two assumptions are valid in the compact case too. In fact we extend the above requirements to the full $\mathcal{A}_{L} \times \mathcal{A}_{R}$ algebra. Although our pool of configuration space observables lies in $\mathcal{A}_{L}$, this is important because $\hat{x}_{R}^{a}$ appear in the phase space algebra too.

Our interest is to construct the quantum mechanical phase spaces of noncommutative manifolds with a nontrivial vielbein, guided by Eq. (3.2). In this process, it is often needed to perform a Weyl ordering and we will explain when this is needed and how it is implemented. We always assume that the quantization is performed along the symplectic structure of the manifold and therefore the commutator of position operators corresponds to the components of the symplectic 2-vector in the curved basis. On the other hand, we do not assume anything for the commutator among the momenta and instead we are going to derive it. According to the above, the notation we use is

$$
\begin{aligned}
{\left[\hat{x}^{a}, \hat{x}^{b}\right] } & =i \theta^{a b}, \\
{\left[\hat{x}^{a}, \hat{p}_{i}\right] } & =i \hbar e^{a}{ }_{i}, \\
{\left[\hat{p}_{i}, \hat{p}_{j}\right] } & =i F_{i j} .
\end{aligned}
$$

When we write $\hat{x}^{a}$ without a subscript, we implicitly mean the left acting operators. The right acting ones will always be indicated explicitly as $\hat{x}_{R}^{a}$, and their commutation relation with the momentum operators should be implemented in the above algebra. This will be done explicitly in the following. Finally, let us recall that this notation is appropriate for the noncompact cases. As we saw in the example of the noncommutative torus, the position operators have to be exponentiated in the compact case. We will denote these exponentiated operators as $X^{a}$, as we did in the previous section.

\subsection{Momentum operators in the presence of a nontrivial frame}

Let us proceed a step further and determine the general properties of the position and momentum operators for an arbitrary symplectic parallelizable manifold in d dimensions. We begin with the noncompact case. We would like to determine the momenta which guarantee that the mixed commutation relation $\left[\hat{x}^{a}, \hat{p}_{i}\right]=e_{i}^{a}$ is satisfied. To this end we consider a similar Ansatz as for the case with trivial frame in Section 2,

$$
\hat{p}_{i}=: c_{i a}\left(\hat{x}_{L}^{b}, \hat{x}_{R}^{b}\right)\left(\hat{x}_{L}^{a}-\hat{x}_{R}^{a}\right):,
$$

with two notable differences than previously. First, we let the quantities $c_{i a}$, which have to be determined, to depend on $\hat{x}^{a}$. For the moment this dependence can be both on the left and right acting operators but we will see that a reduction of this dependence is necessary. The above Ansatz and the $\hat{x}^{a}$ dependence of $c_{i a}$ immediately introduces an ordering issue. This is similar to quantum mechanics, when products of position and momentum operators are encountered. The usual recipe is to introduce a Weyl ordering of the operators, denoted as : : :, such as

$$
: \hat{x} \hat{p}:=\frac{1}{2}(\hat{x} \hat{p}+\hat{p} \hat{x}),
$$

and similarly for higher order ambiguities. Presently, the Weyl ordering means that 9

$$
\hat{p}_{i}=\frac{1}{2}\left(c_{i a} \hat{x}_{L}^{a}+\hat{x}_{L}^{a} c_{i a}-c_{i a} \hat{x}_{R}^{a}-\hat{x}_{R}^{a} c_{i a}\right) .
$$

\footnotetext{
${ }^{9}$ If the $c_{i a}$ 's are higher than linear order, then they are also normal ordered. We refrain from explicitly indicating this in our notation.
} 
Having specified the Ansatz, let us insert it in the ECCRs in order to determine the unknown functions $c_{i a}$ :

$$
\begin{aligned}
i \hbar e_{i}^{b}=\left[\hat{x}^{b}, \hat{p}_{i}\right] & =\frac{1}{2}\left(\hat{x}_{L}^{b} c_{i a} \hat{x}_{L}^{a}+\hat{x}_{L}^{b} \hat{x}_{L}^{a} c_{i a}-\hat{x}_{L}^{b} c_{i a} \hat{x}_{R}^{a}-\hat{x}_{L}^{b} \hat{x}_{R}^{a} c_{i a}-\right. \\
& \left.-c_{i a} \hat{x}_{L}^{a} \hat{x}_{L}^{b}-\hat{x}_{L}^{a} c_{i a} \hat{x}_{L}^{b}+c_{i a} \hat{x}_{R}^{a} \hat{x}_{L}^{b}+\hat{x}_{R}^{a} c_{i a} \hat{x}_{L}^{b}\right) .
\end{aligned}
$$

In order to be able to solve this condition, an assumption on the dependence of $c_{i a}$ has to be made. If we assume that the dependence is on $\hat{x}_{L}^{a}$, the commutation relation $\left[\hat{x}_{L}^{a}, \hat{x}_{R}^{b}\right]=0$ may be used to obtain

$$
i \hbar e_{i}^{b}=\frac{1}{2}\left(\hat{x}_{L}^{b} c_{i a} \hat{x}_{L}^{a}+\hat{x}_{L}^{b} \hat{x}_{L}^{a} c_{i a}-2\left[\hat{x}_{L}^{b}, c_{i a}\right] \hat{x}_{R}^{a}-c_{i a} \hat{x}_{L}^{a} \hat{x}_{L}^{b}-\hat{x}_{L}^{a} c_{i a} \hat{x}_{L}^{b}\right) .
$$

This equation is in general not sufficient to determine $c_{i a}$. The situation is greatly improved by the alternative choice of $c_{i a}$ depending on $\hat{x}_{R}^{a}$. In this case we are led to

$$
i \hbar e_{i}^{b}=c_{i a}\left(\hat{x}_{R}^{c}\right)\left[\hat{x}_{L}^{b}, \hat{x}_{L}^{a}\right]=-i c_{i a}\left(\hat{x}_{R}^{c}\right) \theta^{a b},
$$

which implies

$$
c_{i a}\left(\hat{x}_{R}^{c}\right)=\hbar e_{i}^{b} \omega_{b a} .
$$

It is important to stress that this works only if we assume that the noncommutative frame also depends on the right acting set of operators, which is in full agreement with the discussion and assumptions of Section 2.2. This was up to now not specified but it is forced on us by the computation itself and the consistency of the algebra. Therefore we find that the momenta are given as

$$
\hat{p}_{i}=\hbar: e_{i}^{a} \omega_{a b}\left(\hat{x}_{L}^{b}-\hat{x}_{R}^{b}\right):=\hbar: e^{a}{ }_{i} \omega_{a b}\left[\hat{x}^{b}, \cdot\right]: .
$$

A simple consistency check shows that when the frame is trivialized the momenta of the noncommutative plane are recovered. It is observed that in the presence of a nontrivial frame the momenta are complicated operators. Due to the noncommutativity of the frame, it is difficult to proceed in a general evaluation of the momentum commutator $F_{i j}$ and to present a closed form for the conditions due to the Jacobi identities. These tasks are tractable once the frame is given and the symplectic form is known. This will be the case in the next section.

However, let us examine the momentum commutator under some assumptions, which will prove valid in the next section. First, let us assume that $\omega_{a b}$, and therefore $\theta^{a b}$ too, are constant parameters 11 . Then the momenta are simplified to

$$
\hat{p}_{i}=\hbar \omega_{a b}: e^{a}{ }_{i}\left(\hat{x}_{L}^{b}-\hat{x}_{R}^{b}\right): .
$$

We introduce the following notation

$$
\begin{aligned}
& {\left[e_{i}^{a}, \hat{x}_{L}^{b}\right]=0,} \\
& {\left[e^{a}, \hat{x}_{R}^{b}\right]=K_{i}^{a b},} \\
& {\left[e^{a}{ }_{i}, e^{b}{ }_{j}\right]=L_{i j}^{a b} .}
\end{aligned}
$$

\footnotetext{
${ }^{10} \mathrm{Had}$ we made the additional assumption that $\left[c_{i a}\left(\hat{x}_{L}^{c}\right), \hat{x}_{L}^{b}\right]=0$ in the previous case, we would have arrived at the same expression. However, this route would not lead in general to consistent results.

${ }^{11}$ This might seem strange, since they carry curved indices, but in Section 4 we will show that it is a relevant case.
} 
The first equation is due to the fact that the frame is $\hat{x}_{R}^{a}$-dependent. Moreover, it holds that $L_{i j}^{a b}=-L_{j i}^{b a}$ and we do not assume any other symmetry property for $L_{i j}^{a b}$ or $K_{i}^{a b}$. This allows us to compute the momentum commutator and find the simple expression 12

$$
\left[\hat{p}_{i}, \hat{p}_{j}\right]=\hbar^{2} \omega_{a c} \omega_{b d}\left(L_{i j}^{c d}\left(\hat{x}_{L}^{a}-\hat{x}_{R}^{a}\right)\left(\hat{x}_{L}^{b}-\hat{x}_{R}^{b}\right)-2 K_{[i}^{c b} e_{j]}^{d}\left(\hat{x}_{L}^{a}-\hat{x}_{R}^{a}\right)\right) .
$$

It is clear from the structure that the right hand side contains linear and quadratic terms in $\hat{p}_{i}$ and possibly constant terms too. We express this as

$$
\left[\hat{p}_{i}, \hat{p}_{j}\right]=M_{i j}+N_{i j}^{k} \hat{p}_{k}+P_{i j}^{k l} \hat{p}_{k} \hat{p}_{l}
$$

where $M_{i j}, N_{i j}^{k}, P_{i j}^{k l}$ are parameters antisymmetric in their lower indices, while $P_{i j}^{k l}$ is symmetric in its upper indices. We should stress that a quadratic algebra for the momenta was proven by Madore to be the only consistent choice for matrix algebras [1]. Comparing the last two expressions, we determine the coefficients to be

$$
\begin{aligned}
P_{i j}^{k l} & =e^{k}{ }_{c} e^{l}{ }_{d} L_{[i j]}^{c d}, \\
N_{i j}^{k} & =\hbar \omega_{b d} e^{k}{ }_{c}\left(2 K_{[i}^{c b} e^{d}{ }_{j]}+P_{i j}^{m l}\left(K_{l}^{c b} e_{m}^{d}+K_{(m}^{d b} e_{l)}^{c}\right)\right), \\
M_{i j} & =-\frac{\hbar^{2}}{4} \omega_{a c} \omega_{b d} P_{i j}^{k l} K_{k}^{c a} K_{l}^{d b} .
\end{aligned}
$$

Having determined the general form of $F_{i j}$, the last remaining piece is to examine the commutators of $\hat{x}_{R}^{a}$. These commute with $\hat{x}_{L}^{a}$ and they satisfy the opposite algebra to them (symplectic duality). The only unknown commutator is the one with the momentum operators, which we now compute and we find

$$
\left[\hat{x}_{R}^{a}, \hat{p}_{i}\right]=i \hbar e_{i}^{a}-\hbar \omega_{b c} K_{i}^{b a}\left(\hat{x}_{L}^{c}-\hat{x}_{R}^{c}\right)=i \hbar e_{i}^{a}-e^{k}{ }_{b} K_{i}^{b a} \hat{p}_{k}
$$

Therefore, if the frame is known then the full phase space algebra is uniquely determined.

Before proceeding, let us comment on the compact case too. This proceeds along similar lines that led us from the d-plane to the d-torus, with some additional input due to the $\hat{x}_{R}^{a}$-dependence of the frame. A periodicity condition is imposed, but this time there is a difference between the right acting and the left acting operators. The key to understand which conditions to impose for each set is to appreciate that $\hat{x}_{L}^{a}$ are to be associated to positions (property of e.g. a quantum mechanical particle), while $\hat{x}_{R}^{a}$ are to be associated with the frame or gravitational field and therefore to coordinates (property of space itself). This distinction between position and coordinate suggests that the conditions that compactify the space should be applied to the right acting operators, as

$$
\hat{x}_{R}^{a} \sim \hat{x}_{R}^{a}+2 \pi R^{i} \tau_{i}^{a}\left(\hat{x}_{R}^{b}\right)
$$

with some appropriate $\hat{x}_{R}^{a}$-dependent tensor $\tau_{i}^{a}$ that has to be specified. This tensor is related to $e_{i}^{a}$ but it is not the same as that, as it will become obvious in the next section. On the other hand, the positions, which are associated to the operators $\hat{x}_{L}^{a}$, should be single

\footnotetext{
${ }^{12}$ In order to reach this expression, we assume that $\omega_{a c} \omega_{b d}\left[e_{j}^{d}, K_{i}^{c b}\right]=\omega_{a c} \omega_{b d}\left[\hat{x}_{R}^{b}, L_{i j}^{c d}\right]=0$, which can be checked retrospectively in specific cases.
} 
valued when we return on the same point after traversing a cycle of the compact space. For nontrivial elliptic fibrations, where the cycles are of toroidal nature, this means that the periodicity condition on the left acting operators should be analogous to Eq. (2.15), namely

$$
\hat{x}_{L}^{a} \sim \hat{x}_{L}^{a}+2 \pi R^{i} \delta_{i}^{a} .
$$

This is the correct condition because single valuedness has to be guaranteed along the cycle that is traversed and not along other directions too, since in this process the positions in the other directions might have changed due to the nontrivial fibration structure. Then the position operators are obtained by exponentiation as

$$
X^{a}=e^{\frac{i \hat{x}^{a}}{R^{a}}} .
$$

The momenta are now outer, as in the toroidal case, as well as the operators $\hat{x}_{R}^{a}$ which do not correspond to observables and they do not necessarily have to be exponentiated. The phase space algebra in the compact case turns out to be

$$
\begin{aligned}
X^{a} X^{b} & =e^{-\frac{i \theta^{a b}}{R^{a} R^{b}} X^{b} X^{a}}, \\
\hat{p}_{i} X^{a} & =X^{a}\left(\hat{p}_{i}+\frac{\hbar}{R^{a}} e^{a}{ }_{i}\right),
\end{aligned}
$$

and the rest of the commutators remain the same as in the noncompact case. Similar considerations appeared in Refs. [28,29], although in a different and less general context and without reference to symplectic structures. In particular, in those papers the commutator of either the positions or the momenta is set to zero, which is not a consistent choice in the present context, while Jacobi anomalies appear, which is at odds with our assumptions here.

\section{Quantized phase space of symplectic nilmanifolds}

The main general assumptions that we made are the existence of symplectic structure and parallelizability, as well as the Leibniz rule and the Jacobi identity. Having so far worked on the general case, it is now time to examine whether these assumptions include any nontrivial examples. We already know the trivial ones, which are the d-plane in the noncompact case and the d-torus in the compact case. On the other hand, spheres are completely excluded. The only symplectic sphere, $S^{2}$, is not parallelizable and the only parallelizable spheres, $S^{3}$ and $S^{7}$, are not symplectid 13 .

An interesting class of nontrivial symplectic and parallelizable manifolds is provided by group manifolds based on nilpotent Lie algebras and the associated compact nilmanifolds. Therefore we would like to apply the above formalism to this class of spaces. We are going to work in dimensions 4 and 6 . This is a choice based on the following reasons. First, symplectic manifolds are always even-dimensiona 14 . Second, there are no nilmanifolds in

\footnotetext{
13 Spheres can be quantized with different methods, such as the ones used in Refs. 30 33.

${ }^{14}$ This does not mean that odd-dimensional nilmanifolds cannot be quantized using their symplectic leaves. In Ref. [34] a deformation quantization of the 3-dimensional Heisenberg nilmanifold is presented (see also Ref. [35]).
} 
2D, apart from the trivial one of the 2-torus. Dimension 4 contains only two nontrivial cases of nilmanifolds [36, 37] and they are both symplectic. Symplectic nilmanifolds in 6D are also fully classified [37]. They number 26 cases (or, more precisely, classes) and they may be read off either from Chapter 8, Section III of the book [37] or from the table at the end of Ref. [38]. In this section we construct the quantized phase space of these spaces both in the noncompact and compact cases.

\subsection{Step classification of $4 \mathrm{D}$ and $6 \mathrm{D}$ nilmanifolds}

Nilmanifolds were introduced as simple examples of manifolds which admit symplectic structure but not Kähler structure15. From another point of view, they may be described as nontrivial generalizations of the torus, in the sense that any nilmanifold is an iterated twisted fibration of toroidal fibers over toroidal bases. Employing this point of view, the globally defined basis of the cotangent bundle of a nilmanifold can be obtained in an elegant way by twisting the corresponding one for a flat torus. In particular, consider a nilpotent Lie algebra with structure constants $f_{b c}^{a}$. In accord with commonly used notation we present such an algebra as a d-tuple $(a b, c d, \ldots, y z)$, whence its structure constants are $f_{a b}^{1}, f_{c d}^{2}, \ldots, f_{y z}^{\mathrm{d}}$. Then we form the $(1,1)$ tensor

$$
F=\frac{1}{2} \mu_{(a b)} f_{a b}^{c} x^{b} \mathrm{~d} x^{a} \wedge \partial_{c},
$$

parametrized by constants $\mu_{(a b)}$, where the indices between parentheses are not summed and there is no symmetry property that provides a direct relation between $\mu_{(a b)}$ and $\mu_{(b a)}$. Exponentiating this tensor and acting with it on the basis 1 -forms $\delta_{a}^{i} \mathrm{~d} x^{a}$ of the d-torus we get

$$
e^{i}=\delta^{i}{ }_{a} e^{F} \mathrm{~d} x^{a} .
$$

The action is performed with the standard interior product between vectors and forms. Since we are going to work with nilmanifolds up to dimension six, we use an expanded expression for the frame up to terms which are nonvanishing in such cases, i.e.

$$
\begin{aligned}
e_{a}^{i} & =\delta_{a}^{i}+\frac{1}{2} \kappa_{(a b)} f_{a b}^{i} x^{b}+\frac{1}{8} \kappa_{(b c a d)} f_{b c}^{i} f_{a d}^{b} x^{c} x^{d}+\frac{1}{48} \kappa_{(b c p d a q)} f_{b c}^{i} f_{p d}^{b} f_{a q}^{p} x^{c} x^{d} x^{q}+ \\
& +\frac{1}{384} \kappa_{(b c p d r q a s)} f_{b c}^{i} f_{p d}^{b} f_{r q}^{p} f_{a s}^{r} x^{c} x^{d} x^{q} x^{s} .
\end{aligned}
$$

Note that this expression is more general than a simple expansion of Eq. (4.2), since the constants $\kappa_{(\ldots)}$ in front of each term are now not fixed by lower step terms. No symmetry properties for these constants are assumed. The amount of non-vanishing terms on the right hand side is on a par with the nilpotency step of the underlying Lie algebra. For step 1 only the first term is there, which agrees with the fact that a step 1 nilmanifold is a torus. For step 2 we get the first two terms, since in this case it holds that

$$
f_{b c}^{i} f_{a d}^{b}=0,
$$

even without summation in the index $b$, by the definition of step 2. For step 3, Eq. (4.4) is violated but it holds that

$$
f_{b c}^{i} f_{p d}^{b} f_{a q}^{p}=0,
$$

\footnotetext{
${ }^{15}$ The only Kähler nilmanifold is the d-torus 39 . Moreover, nilmanifolds that do not admit a symplectic structure exist too 38 .
} 
again without summation in the repeated indices. Similarly, for step 4, Eqs. (4.4) and (4.5) are violated but the following relation holds

$$
f_{b c}^{i} f_{p d}^{b} f_{r q}^{p} f_{a s}^{r}=0 .
$$

Finally, the step 5 cases violate all the above relations. Step 6 nilmanifolds do not exist in six dimensions by definition. This is the reason that we stopped the expansion of the general formula to these five terms.

The basis 1-vectors can be determined in the same way. Moreover, the inverse $e_{i}^{a}$ of the frame $e_{a}^{i}$ satisfies

$$
e^{i}{ }_{a} e^{a}{ }_{j}=\delta_{j}^{i} \quad \text { and } \quad e_{a}^{i} e_{i}^{b}=\delta_{a}^{b},
$$

and it is given by the analogous expanded formula

$$
\begin{aligned}
e^{a}{ }_{i} & =\delta_{i}^{a}-\frac{1}{2} \lambda_{(i b)} f_{i b}^{a} x^{b}+\frac{1}{8} \lambda_{(c b i d)} f_{c b}^{a} f_{i d}^{c} x^{b} x^{d}-\frac{1}{48} \lambda_{(c b p d i q)} f_{c b}^{a} f_{p d}^{c} f_{i q}^{p} x^{b} x^{d} x^{q}+ \\
& +\frac{1}{384} \lambda_{(c b p d r q i s)} f_{c b}^{a} f_{p d}^{c} f_{r q}^{p} f_{i s}^{r} x^{b} x^{d} x^{q} x^{s}
\end{aligned}
$$

where

$$
\begin{aligned}
\lambda_{(i b)}= & \kappa_{(i b)}, \\
\lambda_{(c b i d)}= & -\kappa_{(c b i d)}+2 \kappa_{(c b)} \kappa_{(i d)}, \\
\lambda_{(c b p d i q)}= & \kappa_{(c b p d i q)}-3 \kappa_{(p d i q)} \kappa_{(c b)}+6 \kappa_{(c b)} \kappa_{(p d)} \kappa_{(i q)}, \\
\lambda_{(c b p d r q i s)}= & -\kappa_{(c b p d r q i s)}+4\left(\kappa_{(p d r q i s)} \kappa_{(c b)}+\kappa_{(c b p d r q)} \kappa_{(i s)}\right)+6 \kappa_{(c b p d)} \kappa_{(r q i s)}- \\
& -12\left(\kappa_{(c b p q)} \kappa_{(r q)} \kappa_{(i s)}+\kappa_{(r q i s)} \kappa_{(p q)} \kappa_{(c b)}\right)+24 \kappa_{(c b)} \kappa_{(p d)} \kappa_{(r q)} \kappa_{(i s)} .
\end{aligned}
$$

Let us turn our attention to the parameters $\kappa_{(\ldots)}$ that were introduced. Clearly, they are not arbitrary since they are constrained by the Maurer-Cartan equations. It is easy to determine this constraint if we focus on the step 2 case, where

$$
e^{i}{ }_{a}=\delta_{a}^{i}+\frac{1}{2} \kappa_{(a b)} f_{a b}^{i} x^{b} .
$$

We compute

$$
\begin{aligned}
-\frac{1}{2} f_{j k}^{i} e^{j k} & =\mathrm{d} e^{i}=\mathrm{d}\left(e^{i}{ }_{a} \mathrm{~d} x^{a}\right)=\partial_{b} e^{i}{ }_{a} \mathrm{~d} x^{b a}=e^{b}{ }_{j} e_{k}^{a} \partial_{b} e^{i}{ }_{a} e^{j k} \\
& \Rightarrow f_{j k}^{i}=e^{a}{ }_{j} e^{b}{ }_{k}\left(\partial_{b} e^{i}{ }_{a}-\partial_{a} e^{i}{ }_{b}\right) \\
& \Rightarrow \kappa_{(a b)}+\kappa_{(b a)}=2 .
\end{aligned}
$$

The most symmetric choice would be $\kappa_{(a b)}=\kappa_{(b a)}=1$, which was used for example in Ref. [29]. However, this is not the choice we make in the present paper and there is a very good reason for this, related to the symplectic structures on the nilmanifolds. We return to this immediately after we discuss these structures.

The classification of nilpotent (but not solvable) Lie algebras in four dimensions can be found in Ref. [36]. There are only three cases to consider. The first is the 4-torus, which is step 1 and symplectic. As we already mentioned, it is a rather degenerate case, in the sense that unlike all the other nilmanifolds, the torus is a Kähler manifold. Moreover, it is a flat space. Its symplectic 2 -form can be chosen to be $\omega=e^{12}+e^{34}$, with $e^{i}=\delta_{a}^{i} \mathrm{~d} x^{a}$. We will 
not discuss it further since it was already discussed in Section 2 in any dimension. The other two cases are given by $(0,0,0,12)$ and $(0,0,42,12)$. The first one is a toroidal extension of the Heisenberg algebra in dimension three and it has nilpotency step 2. The second one has nilpotency step 3 , since it clearly contains the non-zero second order commutator of algebra generators $\left[T_{2},\left[\left[T_{1}, T_{2}\right]\right]=-T_{3}\right.$, or equivalently the non-vanishing quantity $f_{42}^{3} f_{12}^{4}$, but no third order non-vanishing commutator. Both cases admit a non-degenerate symplectic structure. All these are summarized in the following table:

$\begin{array}{|ccc|}\begin{array}{c}\text { Class } \\ (0,0,0,12)\end{array} & \frac{\text { Step }}{2} & \frac{\text { Symplectic form }}{e^{14}+e^{23}} \\ (0,0,42,12) & 3 & e^{14}+e^{23}\end{array}$

Table 1: Nontrivial symplectic nilmanifolds in 4D.

We move on to $6 \mathrm{D}$, where there are 26 classes of symplectic nilmanifolds. We would like to sub-classify them according to their nilpotency step. As before, the 6-torus is the only one step 1 nilmanifold. In the following four tables we present the step 2,3,4 and $56 \mathrm{D}$ symplectic nilmanifolds along with their symplectic 2 -form, the latter taken from the single table of Ref. [38.

\begin{tabular}{|cc|}
\hline$\underline{\text { Class }}$ & $\frac{\text { Symplectic form }}{e^{16}+e^{23}+e^{45}}$ \\
$(0,0,0,0,0,12)$ & $e^{16}+e^{25}+e^{34}$ \\
$(0,0,0,0,13+42,14+23)$ & $e^{16}+e^{25}+e^{34}$ \\
$(0,0,0,0,12,13)$ & $e^{15}+e^{36}+e^{24}$ \\
$(0,0,0,0,12,34)$ & $e^{13}+e^{26}+e^{45}$ \\
$(0,0,0,0,12,14+23)$ & $e^{15}+e^{24}+e^{36}$ \\
\hline
\end{tabular}

Table 2: Step 2 symplectic nilmanifolds in 6D.

\begin{tabular}{|cc|}
\hline$\underline{\text { Class }}$ & Symplectic form \\
$(0,0,0,0,12,14+25)$ & $e^{13}+e^{26}+e^{45}$ \\
$(0,0,0,0,12,15)$ & $e^{16}+e^{25}+e^{34}$ \\
$(0,0,0,12,14+23,13+42)$ & $e^{15}+2 e^{26}+e^{34}$ \\
$(0,0,0,12,14,13+42)$ & $e^{15}+e^{26}+e^{34}$ \\
$(0,0,0,12,14,23+24)$ & $e^{16}-e^{34}+e^{25}$ \\
$(0,0,0,12,13,14)$ & $e^{16}+e^{24}+e^{35}$ \\
$(0,0,0,12,13,24)$ & $e^{26}+e^{14}+e^{35}$ \\
$(0,0,0,12,13,14+23)$ & $e^{16}-2 e^{34}-e^{25}$ \\
\hline
\end{tabular}

Table 3: Step 3 symplectic nilmanifolds in 6D. 


\begin{tabular}{|cc|}
\hline$\underline{\text { Class }}$ & $\frac{\text { Symplectic form }}{e^{16}+e^{35}+e^{24}}$ \\
$(0,0,0,12,14-23,15+34)$ & $e^{13}+e^{26}-e^{45}$ \\
$(0,0,0,12,14,15)$ & $e^{13}+e^{26}-e^{45}$ \\
$(0,0,0,12,14,15+24)$ & $e^{13}+e^{26}-e^{45}$ \\
$(0,0,0,12,14,15+23+24)$ & $e^{13}+e^{26}-e^{45}$ \\
$(0,0,0,12,14,23+15)$ & $e^{15}+e^{24}+e^{34}-e^{26}$ \\
$(0,0,12,13,23,14)$ & $e^{15}+e^{24}-e^{35}+e^{16}$ \\
$(0,0,12,13,23,14-25)$ & $e^{15}+e^{24}+e^{35}+e^{16}$ \\
$(0,0,12,13,23,14+25)$ &
\end{tabular}

Table 4: Step 4 symplectic nilmanifolds in 6D.

\begin{tabular}{|cc|}
\hline Class & $\frac{\text { Symplectic form }}{e^{16}+e^{34}-e^{25}}$ \\
$(0,0,12,13,14,15)$ & $e^{16}+e^{34}+e^{24}-e^{25}$ \\
$(0,0,12,13,14,15+23)$ & $e^{16}+2 e^{34}-e^{25}$ \\
$(0,0,12,13,14+23,15+24)$ &
\end{tabular}

Table 5: Step 5 symplectic nilmanifolds in 6D.

The above five tables contain 27 cases where we can apply the results of Section 3. It is interesting and welcome that a lot of diversity is exhibited, since there are indeed cases up to step 5 which do admit symplectic structure.

\subsection{Determining the phase space algebra}

It is obvious from the above tables that in the flat basis of $e^{i}$, the components of the symplectic 2-form and the corresponding 2-vector are constant. However, this is not in general true for the symplectic 2-form in an arbitrary curved basis. Let us discuss how and when the curved basis components $\omega_{a b}$ and $\theta^{a b}$ can be constant as well. This is essentially the reason that we introduced the constants $\kappa_{(\ldots)}$ previously, instead of making the symmetric choice for them.

We propose the following:

Proposition: For 4D and 6D symplectic nilmanifolds whose symplectic structure has the same form as the corresponding torus, there exists a coordinate system, specified by a choice of parameters $\kappa_{(\ldots)}$, such that the components $\omega_{a b}\left(\theta^{a b}\right)$ of the symplectic 2-form (2-vector) in the curved basis are constant and equal to the flat components $\omega_{i j}\left(\theta^{i j}\right)$.

Proof: It is a straightforward task to determine the form of $\kappa_{(\ldots)}$ for each case separately and the corresponding frame $e_{a}^{i}$ that delivers the required result. The full list of results for the frame $e_{a}^{i}$ appears in the tables of the Appendix and proves the proposition.

This proposition shows that all but four symplectic nilmanifolds satisfy the assumption that led to Eqs. (3.28) and (3.29) with coefficients (3.30)-(3.32), which are central for our 
purposes 16 . This in turn means that the full phase space algebra can be determined in a closed form for all these cases. To this end, the momentum commutator has to be fully determined and this will be the case once we compute the quantities $K_{i}^{a b}$ and $L_{i j}^{a b}$, defined in Eqs. (3.26) and (3.27). This is possible because the frame is known explicitly in all cases.

Let us work out in full detail the step 2 case. We compute

$$
K_{i}^{a b}=-\frac{i}{2} \kappa_{(i c)} f_{i c}^{a} \theta^{b c}
$$

and

$$
L_{i j}^{a b}=\frac{i}{4} \kappa_{(i c)} \kappa_{(j d)} f_{i c}^{a} f_{j d}^{b} \theta^{d c}
$$

We directly obtain that

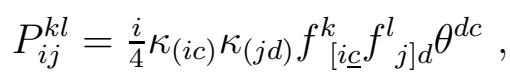

due to Eq. (4.4), where the underlined index is excluded from the antisymmetrization. The same equation implies also that

$$
P_{i j}^{k l} K_{l}^{a b}=0 .
$$

Then the Eqs. (3.31) and (3.32) give

$$
M_{i j}=0, \quad N_{i j}^{k}=-i \hbar f_{i j}^{k},
$$

where Eq. (4.9) was used. Let us mention once more that the constants $\kappa_{(i a)}$ are known for each case, since they determine the frame components that guarantee the constancy of $\theta^{a b}$. This leads to the following phase space algebra in the step 2 case:

$$
\left[\hat{x}^{a}, \hat{x}^{b}\right]=i \theta^{a b}, \quad\left[\hat{x}^{a}, \hat{p}_{i}\right]=i \hbar e_{i}^{a}, \quad\left[\hat{p}_{i}, \hat{p}_{j}\right]=-i \hbar f_{i j}^{k} \hat{p}_{k}+\frac{i}{4} \kappa_{(i c)} \kappa_{(j d)} f_{[i \underline{c}}^{k} f_{j] d}^{l} \theta^{d c} \hat{p}_{k} \hat{p}_{l} .
$$

Additionally,

$$
\left[\hat{x}_{R}^{a}, \hat{x}_{R}^{b}\right]=-i \theta^{a b}, \quad\left[\hat{x}_{R}^{a}, \hat{p}_{i}\right]=i \hbar e_{i}^{a}+\frac{i}{2} \kappa_{(i c)} f_{i c}^{k} \theta^{a c} \hat{p}_{k} .
$$

The Jacobi identities have to be satisfied as well. Nontrivial ones include

$$
\operatorname{Jac}\left(\hat{p}_{i}, \hat{p}_{j}, \hat{p}_{k}\right)=0
$$

which is satisfied due to Eq. (4.4), and

$$
\operatorname{Jac}\left(\hat{p}_{i}, \hat{p}_{j}, \hat{x}^{a}\right)=0 \Rightarrow\left[e_{i}^{a}, \hat{p}_{j}\right]-\left[e^{a}, \hat{p}_{i}\right]=i \hbar f_{i j}^{a}-2 P_{i j}^{k l} e_{(k}^{a} \hat{p}_{l)} .
$$

The latter is not obvious, but we have checked that it is satisfied in all step 2 cases, using the data of the Appendix. In the following, it will be examined in some explicit examples too, also for higher steps. The same holds for the Jacobi identities that involve $\hat{x}_{R}^{a}$.

We point out that the resemblance to the planar case is exhausted in the constancy of the parameters $\theta^{a b}$. A comparison of Eqs. (4.15) and (4.16) with the corresponding ones of the planar case shows that the two algebras are very different.

\footnotetext{
${ }^{16}$ The four cases that are not covered are indicated accordingly in the tables of the Appendix and they are three step 4 cases and one step 5. They can also be found in Tables 4 and 5 as the ones whose symplectic form involves four summands.
} 


\subsection{Remarks on the compact case}

As a final task, we examine the compact case. This is captured by the general expressions (3.37) and (3.38), where the parameters $\theta^{a b}$ and $e^{a}{ }_{i}$ are explicitly known for the cases at hand. Here we would like to include some additional remarks on the compactification of the group manifold, in order to make clear why the exponentiated coordinates are given by Eq. (3.36) in the nontoroidal case and they do not have to be modified from the toroidal ones of Eq. (2.19).

Consider the simplest case of step 2 nilmanifolds, since the higher step cases are just direct generalizations of the procedure. Here we work on the classical manifold. The globally well defined 1-forms are

$$
e^{i}=\left(\delta_{a}^{i}+\frac{1}{2} \kappa_{(a b)} f_{a b}^{i} x^{b}\right) \mathrm{d} x^{a} .
$$

Consider shifting the coordinate $x^{b} \rightarrow x^{b}+2 \pi \delta^{b}{ }_{i} R^{i}$. Then

$$
e^{i} \rightarrow e_{a}^{i} \mathrm{~d} x^{\prime a}+\frac{1}{2} \kappa_{(a b)} f_{a b}^{i} \delta_{i}^{b} 2 \pi R^{i} \mathrm{~d} x^{\prime a} .
$$

Since the frame is global, it should remain invariant under this shift and this means that $x^{a}$ cannot remain the same. Indeed we observe that they have to change to

$$
x^{\prime a}=x^{a}-\frac{1}{2} \kappa_{(b i)} f_{b i}^{a} x^{b} 2 \pi R^{i} .
$$

Then using again Eq. (4.4), we find that $e^{i} \rightarrow e^{i}$, as it should. Evidently, although the 1 -forms $e^{i}$ are globally well defined, this is not true for the coordinates $x^{a}$. On the contrary, on a torus it is possible to define global coordinates besides global 1-forms. In other words, a shift around a cycle in the toroidal case leads back to the same point and therefore the position in every direction has to be exactly the same, which is achieved with exponentiation. In the nilmanifold case, a shift around a cycle associated with the topologically nontrivial fibration structure of the manifold leads to the same point in the direction of the cycle, but to a different point in the orthogonal directions, since the fibered tori have changed geometrically according to the nontrivial twist. This means that the position has to be single valued in the direction of the shift, but in the fiber directions this position will naturally change, exactly because the test particle never returned to the exact same position that it had before the shift in those directions. This result indicates that the single-valuedness along the shifted direction is taken care of by exponentiation, exactly as for the toroidal case. On the other hand, the change of position due to the twist is encoded in the commutation relation between the momenta (which are translation operators and as such they correspond to the generators of the shift) and the positions, as in Eq. (3.38).

As we explained in Section 3.2, the compactification of the nilmanifold in the noncommutative case requires a stronger periodicity condition on the operators $\hat{x}_{R}^{a}$ that provide the frame dependence. This is given by Eq. (3.34). Eq. (4.21) suggests that the overall periodic shift in the $\hat{x}_{R}^{a}$ is

$$
\hat{x}_{R}^{a} \sim \hat{x}_{R}^{a}+2 \pi R^{i}\left(\delta^{a}{ }_{i}-\frac{1}{2} \kappa_{(b i)} f_{b i}^{a} \hat{x}_{R}^{b}\right) .
$$

This gives the dual noncommutative frame

$$
\tau_{i}^{a}=\delta_{i}^{a}-\frac{1}{2} \kappa_{(b i)} f_{b i}^{a} \hat{x}_{R}^{b}
$$


for the step 2 case and we can see that this is different than $e^{a}{ }_{i}$, since $\kappa_{(i a)}$ has no symmetry property. Its inverse is

$$
\tau_{a}^{i}=\delta_{a}^{i}+\frac{1}{2} \kappa_{(b a)} f_{b a}^{i} \hat{x}_{R}^{b} .
$$

Although these considerations seem very different than the simple toroidal case, it should be reminded that in the case of the compactification of the plane one also encounters two different tori dual to each other, one associated to the periodicity condition (2.15) and one to the algebraic relation (2.21). The situation is analogous here, where there are two nilmanifolds, one associated to the inverse frame $e^{a}{ }_{i}$ in Eq. (3.38) and one to the dual inverse frame $\tau_{i}^{a}$ of the periodicity condition (3.34).

\section{Benchmark examples of quantized phase spaces}

\subsection{Dimension 4}

Let us now proceed and apply the general results in some benchmark cases from the tables of symplectic nilmanifolds, beginning with dimension four. There are only two cases, so we examine both of them.

Step 2: $(\mathbf{0 , 0 , 0 , 1 2 )}$. In the present case the only non-vanishing structure constant is the $f_{12}^{4}=-f_{21}^{4}=1$. The basis of the cotangent bundle is

$$
e^{i}=\mathrm{d} x^{i}, i=1,2,3, \quad e^{4}=\mathrm{d} x^{4}+x^{2} \mathrm{~d} x^{1} .
$$

In other words, $\kappa_{(12)}=2$ and $\kappa_{(21)}=0$. All the other parameters vanish. It holds that

$$
\mathrm{d} e^{4}=-e^{12},
$$

which is essentially the Maurer-Cartan equation. The symplectic structure in the natural basis is specified by the 2 -form

$$
\omega=e^{14}+e^{23}
$$

and it is easily confirmed that it is closed and non-degenerate. Moreover, it is easily confirmed that

$$
e^{14}=\mathrm{d} x^{14} \quad \text { and } \quad e^{23}=\mathrm{d} x^{23} .
$$

The dual basis is spanned by the vectors

$$
\theta_{1}=\partial_{1}-x^{2} \partial_{4}, \quad \theta_{i}=\partial_{i}, i=2,3,4 .
$$

The symplectic 2-vector is 17

$$
\theta=\theta_{14}+\theta_{23}=\partial_{14}+\partial_{23} .
$$

Then we set

$$
\left[\hat{x}^{1}, \hat{x}^{4}\right]=i \quad \text { and } \quad\left[\hat{x}^{2}, \hat{x}^{3}\right]=i .
$$

\footnotetext{
${ }^{17}$ Here $\theta_{14}=\theta_{1} \wedge \theta_{4}$ and it should not be confused with the constant component $\theta^{14}=1$.
} 
The fact that the only non-vanishing off-diagonal element of $e^{i}{ }_{a}$ is the $e^{4}{ }_{1}=x^{2}$ means that for its inverse $e_{i}^{a}$ the corresponding component is equal to $-x^{2}$ and therefore the noncommutative frame will have $e^{4}{ }_{1}=-\hat{x}_{R}^{2}$, according to the analysis of Section 318 . This means that

$$
\left[\hat{x}^{4}, \hat{p}_{1}\right]=-i \hbar \hat{x}_{R}^{2} .
$$

The momenta are determined by Eq. (3.25) and they turn out to be

$$
\begin{aligned}
& \hat{p}_{1}=\hbar\left(\left[\hat{x}^{4}, \cdot\right]+\hat{x}_{R}^{2}\left[\hat{x}^{1}, \cdot\right]\right), \\
& \hat{p}_{2}=\hbar\left[\hat{x}^{3}, \cdot\right] \\
& \hat{p}_{3}=-\hbar\left[\hat{x}^{2}, \cdot\right] \\
& \hat{p}_{4}=-\hbar\left[\hat{x}^{1}, \cdot\right] .
\end{aligned}
$$

We observe that no ordering is necessary in any of the four momentum operators. Computing the commutators of the momenta, we find that the only non-vanishing one is

$$
\left[\hat{p}_{1}, \hat{p}_{2}\right]=-i \hbar \hat{p}_{4}
$$

in accord with the general results of the previous section for the quantity $N_{i j}{ }^{k}$. For the right acting operators we find the non-vanishing off-diagonal commutators

$$
\left[\hat{x}_{R}^{3}, \hat{p}_{1}\right]=-i \hat{p}_{4}, \quad\left[\hat{x}_{R}^{4}, \hat{p}_{1}\right]=-i \hbar x_{R}^{2} .
$$

It is a straightforward task to show that all the Jacobi identities are satisfied identically. Let us now consider the compact case. From Eq. (5.1) we get that for the classical manifold

$$
x^{2} \rightarrow x^{2}+2 \pi R^{2} \quad \Rightarrow \quad x^{4} \rightarrow x^{4}-2 \pi R^{2} x^{1},
$$

which means that the periodicity condition (3.34) in the noncommutative case involves the off-diagonal component

$$
\tau_{2}^{4}=-\hat{x}_{R}^{1}
$$

According to Eq. (3.36) the position operators are given by

$$
X^{a}=e^{\frac{i}{R^{a}} \hat{x}^{a}}, a=1,2,3,4 .
$$

Then we can find the phase space algebra in the compact case, which is

$$
\begin{aligned}
X^{1} X^{4} & =e^{-\frac{i}{R^{1} R^{4}} X^{4} X^{1}}, \quad X^{2} X^{3}=e^{-\frac{i}{R^{2} R^{3}} X^{3} X^{2}}, \\
\hat{p}_{1} X^{4} & =X^{4}\left(\hat{p}_{1}-\frac{\hbar}{R^{4}} \hat{x}_{R}^{2}\right)
\end{aligned}
$$

plus the diagonal mixed relations and the momentum commutators which were already written down above.

\footnotetext{
${ }^{18}$ This can be directly derived from Eq. (4.8), or one can write the frame as a matrix and find its inverse, in particular

$$
e^{i}{ }_{a}=\left(\begin{array}{cccc}
1 & 0 & 0 & 0 \\
0 & 1 & 0 & 0 \\
0 & 0 & 1 & 0 \\
x^{2} & 0 & 0 & 1
\end{array}\right) \Rightarrow e_{i}^{a}=\left(\begin{array}{cccc}
1 & 0 & 0 & 0 \\
0 & 1 & 0 & 0 \\
0 & 0 & 1 & 0 \\
-x^{2} & 0 & 0 & 1
\end{array}\right),
$$
}

for the present example. 
Step 3: $\left(\mathbf{0 , 0 , 4 2 , 1 2 )}\right.$. In this case the non-vanishing structure constants are $f_{42}^{3}=$ $f_{12}^{4}=1$. The basis of the cotangent bundle is taken to be

$$
e^{i}=\mathrm{d} x^{i}, i=1,2, \quad e^{3}=\mathrm{d} x^{3}-x^{4} \mathrm{~d} x^{2}-x^{1} x^{2} \mathrm{~d} x^{2}, \quad e^{4}=\mathrm{d} x^{4}+x^{2} \mathrm{~d} x^{1} .
$$

This time the only non-vanishing parameters are $\kappa_{(24)}=\kappa_{(12)}=2$ and $\kappa_{(4221)}=8$. The dual vectors are easily found,

$$
\theta_{1}=\partial_{1}-x^{2} \partial_{4}, \quad \theta_{2}=\partial_{2}+\left(x^{4}+x^{2} x^{1}\right) \partial_{3}, \quad \theta_{i}=\partial_{i}, i=3,4 .
$$

The symplectic 2 -form is

$$
\omega=e^{14}+e^{23}=\mathrm{d} x^{14}+\mathrm{d} x^{23},
$$

while the corresponding 2 -vector is

$$
\theta=\theta_{14}+\theta_{23}=\partial_{14}+\partial_{23}
$$

and we set again

$$
\left[\hat{x}^{1}, \hat{x}^{4}\right]=i \quad \text { and } \quad\left[\hat{x}^{2}, \hat{x}^{3}\right]=i .
$$

The only non-vanishing off-diagonal elements of $e^{a}{ }_{i}$ are the $e^{3}{ }_{2}=-\left(x^{4}+x^{2} x^{1}\right)$ and $e^{4}{ }_{1}=$ $x^{2}$; the noncommutative frame has $e_{2}^{3}=\hat{x}_{R}^{4}+\hat{x}_{R}^{1} \hat{x}_{R}^{2}$ and $e^{4}{ }_{1}=-\hat{x}_{R}^{2}$, which sets the commutation relations

$$
\left[\hat{x}^{3}, \hat{p}_{2}\right]=i \hbar\left(\hat{x}_{R}^{4}+\hat{x}_{R}^{1} \hat{x}_{R}^{2}\right), \quad\left[\hat{x}^{4}, \hat{p}_{1}\right]=-i \hbar \hat{x}_{R}^{2} .
$$

The momenta are again found using Eq. (3.25) and they turn out to be

$$
\begin{aligned}
& \hat{p}_{1}=\hbar\left(\left[\hat{x}^{4}, \cdot\right]+\hat{x}_{R}^{2}\left[\hat{x}^{1}, \cdot\right]\right) \\
& \hat{p}_{2}=\hbar\left(\left[\hat{x}^{3}, \cdot\right]-\left(\hat{x}_{R}^{4}+\hat{x}_{R}^{1} \hat{x}_{R}^{2}\right)\left[\hat{x}^{2}, \cdot\right]\right), \\
& \hat{p}_{3}=-\hbar\left[\hat{x}^{2}, \cdot\right] \\
& \hat{p}_{4}=-\hbar\left[\hat{x}^{1}, \cdot\right] .
\end{aligned}
$$

The non-vanishing momentum commutators are

$$
\left[\hat{p}_{2}, \hat{p}_{4}\right]=i \hbar \hat{p}_{3}, \quad\left[\hat{p}_{1}, \hat{p}_{2}\right]=-i \hbar \hat{p}_{4}
$$

Once more, all the Jacobi identities are satisfied. The least trivial one is

$$
\begin{aligned}
\operatorname{Jac}\left(\hat{p}_{1}, \hat{p}_{2}, \hat{x}^{3}\right) & =\left[\left[\hat{p}_{1}, \hat{p}_{2}\right], \hat{x}^{3}\right]+\left[\left[\hat{x}^{3}, \hat{p}_{1}\right], \hat{p}_{2}\right]+\left[\left[\hat{p}_{2}, \hat{x}^{3}\right], \hat{p}_{1}\right]= \\
& =0+0-i \hbar\left[\hat{x}_{R}^{4}+\hat{x}_{R}^{1} \hat{x}_{R}^{2}, \hat{p}_{1}\right]= \\
& =\hbar^{2} \hat{x}_{R}^{2}-\hbar^{2} \hat{x}_{R}^{2}=0
\end{aligned}
$$

where we used the fact that a direct computation gives $\left[\hat{x}_{R}^{4}, \hat{p}_{1}\right]=-i \hbar x_{R}^{2}$. The rest of the commutators involving $\hat{x}_{R}^{a}$ are

$\left[\hat{x}_{R}^{3}, \hat{p}_{1}\right]=-i \hat{p}_{4}, \quad\left[\hat{x}_{R}^{3}, \hat{p}_{2}\right]=i \hbar\left(\hat{x}_{R}^{4}+\hat{x}_{R}^{1} \hat{x}_{R}^{2}\right)+i \hat{x}_{R}^{1} \hat{p}_{3}, \quad\left[\hat{x}_{R}^{1}, \hat{p}_{2}\right]=-i \hat{p}_{3}, \quad\left[\hat{x}_{R}^{4}, \hat{p}_{2}\right]=i \hat{x}_{R}^{2} \hat{p}_{3}$. 
For the compact case, the frame (5.12) suggests the nontrivial shifts

$$
\begin{aligned}
& x^{4} \rightarrow x^{4}+2 \pi R^{4} \quad \Rightarrow \quad x^{3} \rightarrow x^{3}+2 \pi R^{4} x^{2}, \\
& x^{2} \rightarrow x^{2}+2 \pi R^{2} \quad \Rightarrow \quad x^{4} \rightarrow x^{4}-2 \pi R^{2} x^{1}, \\
& x^{1} \rightarrow x^{1}+2 \pi R^{1} \quad \Rightarrow \quad x^{3} \rightarrow x^{3}+\frac{2 \pi R^{1}}{2}\left(x^{2}\right)^{2},
\end{aligned}
$$

in the classical case. This means that in the noncommutative case the off-diagonal $\tau_{i}^{a}$ components are

$$
\tau_{4}^{3}=\hat{x}_{R}^{2}, \quad \tau_{1}^{3}=\frac{1}{2}\left(\hat{x}_{R}^{2}\right)^{2}, \quad \tau_{2}^{4}=-\hat{x}_{R}^{1} .
$$

Then it is straightforward to compute the relations among the $X^{a}$ and $\hat{p}_{i}$, which turn out to be (we write down only the nontrivial ones)

$$
\begin{aligned}
X^{1} X^{4} & =e^{-\frac{i}{R^{1} R^{4}} X^{4} X^{1}}, \quad X^{2} X^{3}=e^{-\frac{i}{R^{2} R^{3}} X^{3} X^{2}} \\
\hat{p}_{2} X^{3} & =X^{3}\left(\hat{p}_{2}-\frac{\hbar}{R^{3}}\left(\hat{x}^{4}+\hat{x}^{1} \hat{x}^{2}\right)_{R}\right), \quad \hat{p}_{1} X^{4}=X^{4}\left(\hat{p}_{1}-\frac{\hbar}{R^{4}} \hat{x}_{R}^{2}\right) .
\end{aligned}
$$

\subsection{Dimension 6}

In six dimensions there are many cases and we will not present all of them in detail. The phase spaces for each case can be reconstructed with the data we collect in the Appendix. Here we would like to examine in detail two representative examples, one step 2 and one step 5, which have properties that are absent in the 4D cases.

Step 2: $(\mathbf{0}, \mathbf{0}, \mathbf{0}, \mathbf{0}, \mathbf{1 3}+\mathbf{4 2 , 1 4 + 2 3 )}$. We pick this representative case of step 2 nilmanifold in six dimensions because its momentum commutator contains a quadratic term, i.e. $P_{i j}^{k l} \neq$ 0 , which did not happen in the 4D cases.

According to the corresponding entry in the Appendix, the basis 1-forms are

$$
e^{i}=\mathrm{d} x^{i}, i=1, \ldots, 4, \quad e^{5}=\mathrm{d} x^{5}+x^{3} \mathrm{~d} x^{1}-x^{4} \mathrm{~d} x^{2}, \quad e^{6}=\mathrm{d} x^{6}+x^{4} \mathrm{~d} x^{1}+x^{3} \mathrm{~d} x^{2} .
$$

It is immediately confirmed that for the symplectic 2-form it holds that

$$
e^{16}+e^{25}+e^{34}=\mathrm{d} x^{16}+\mathrm{d} x^{25}+\mathrm{d} x^{34},
$$

as it is required in our analysis. The dual vectors are

$$
\theta_{1}=\partial_{1}-x^{3} \partial_{5}-x^{4} \partial_{6}, \quad \theta_{2}=\partial_{2}+x^{4} \partial_{5}-x^{3} \partial_{6}, \quad \theta_{i}=\partial_{i}, i=3, \ldots, 6,
$$

and obviously

$$
\theta=\theta_{16}+\theta_{25}+\theta_{34}=\partial_{16}+\partial_{25}+\partial_{34} .
$$

Then the non-vanishing position commutators are

$$
\left[\hat{x}^{1}, \hat{x}^{6}\right]=\left[\hat{x}^{2}, \hat{x}^{5}\right]=\left[\hat{x}^{3}, \hat{x}^{4}\right]=i .
$$

The nonconstant inverse frame components read as

$$
e^{5}{ }_{1}=-\hat{x}_{R}^{3}, \quad e^{5}{ }_{2}=\hat{x}_{R}^{4}, \quad e^{6}{ }_{1}=-\hat{x}_{R}^{4}, \quad e^{6}{ }_{2}=-\hat{x}_{R}^{3},
$$


leading to the mixed commutators

$$
\left[\hat{x}^{5}, \hat{p}_{1}\right]=\left[\hat{x}^{6}, \hat{p}_{2}\right]=-i \hbar \hat{x}_{R}^{3}, \quad\left[\hat{x}^{5}, \hat{p}_{2}\right]=-\left[\hat{x}^{6}, \hat{p}_{1}\right]=i \hbar \hat{x}_{R}^{4} .
$$

Eq. (3.25) yields the momenta

$$
\begin{aligned}
& \hat{p}_{1}=\hbar\left(\left[\hat{x}^{6}, \cdot\right]+\hat{x}_{R}^{3}\left[\hat{x}^{2}, \cdot\right]+\hat{x}_{R}^{4}\left[\hat{x}^{1}, \cdot\right]\right), \\
& \hat{p}_{2}=\hbar\left(\left[\hat{x}^{5}, \cdot\right]-\hat{x}_{R}^{4}\left[\hat{x}^{2}, \cdot\right]+\hat{x}_{R}^{3}\left[\hat{x}^{1}, \cdot\right]\right), \\
& \hat{p}_{3}=\hbar\left[\hat{x}^{4}, \cdot\right], \quad \hat{p}_{4}=-\hbar\left[\hat{x}^{3}, \cdot\right] \\
& \hat{p}_{5}=-\hbar\left[\hat{x}^{2}, \cdot\right], \quad \hat{p}_{6}=-\hbar\left[\hat{x}^{1}, \cdot\right] .
\end{aligned}
$$

Their commutation relations can be determined by direct computation and the nonvanishing ones are

$$
\begin{aligned}
& {\left[\hat{p}_{1}, \hat{p}_{3}\right]=\left[\hat{p}_{4}, \hat{p}_{2}\right]=-i \hbar \hat{p}_{5}} \\
& {\left[\hat{p}_{1}, \hat{p}_{4}\right]=\left[\hat{p}_{2}, \hat{p}_{3}\right]=-i \hbar \hat{p}_{6}} \\
& {\left[\hat{p}_{1}, \hat{p}_{2}\right]=i\left(\hat{p}_{5}\right)^{2}+i\left(\hat{p}_{6}\right)^{2} .}
\end{aligned}
$$

We observe a quadratic commutator in the last line, which is essentially due to the nonvanishing $P_{12}^{55}=P_{12}^{66}=i$ parameters, as listed in the Appendix.

The Jacobi identities involve a nontrivial cancellation. This appears in the identities $\operatorname{Jac}\left(\hat{p}_{1}, \hat{p}_{2}, \hat{x}^{5}\right)=0$ and $\operatorname{Jac}\left(\hat{p}_{1}, \hat{p}_{2}, \hat{x}_{R}^{5}\right)=0$. Let us go through the first, since the second works the same way. We compute

$$
\begin{aligned}
& {\left[\left[\hat{p}_{1}, \hat{p}_{2}\right], \hat{x}^{5}\right]=\left[i\left(\hat{p}_{5}\right)^{2}+i\left(\hat{p}_{6}\right)^{2}, \hat{x}^{5}\right]=i\left[\left(\hat{p}_{5}\right)^{2}, \hat{x}^{5}\right]=2 \hbar \hat{p}_{5},} \\
& {\left[\left[\hat{x}^{5}, \hat{p}_{1}\right], \hat{p}_{2}\right]=\left[-i \hbar \hat{x}_{R}^{3}, \hat{p}_{2}\right]=-\hbar \hat{p}_{5},} \\
& {\left[\left[\hat{p}_{2}, \hat{x}^{5}\right], \hat{p}_{1}\right]=\left[-i \hbar \hat{x}_{R}^{4}, \hat{p}_{1}\right]=-\hbar \hat{p}_{5},}
\end{aligned}
$$

where we directly computed and used that $\left[\hat{x}_{R}^{3}, \hat{p}_{2}\right]=\left[\hat{x}_{R}^{4}, \hat{p}_{1}\right]=i \hat{p}_{5}$. Adding up the three terms we indeed confirm that the Jacobi identity is satisfied. Moreover, the rest of the nontrivial commutators involving $\hat{x}_{R}^{a}$ are

$\left[\hat{x}_{R}^{3}, \hat{p}_{1}\right]=-\left[\hat{x}_{R}^{4}, \hat{p}_{2}\right]=i \hat{p}_{6}, \quad\left[\hat{x}_{R}^{5}, \hat{p}_{1}\right]=\left[\hat{x}_{R}^{6}, \hat{p}_{2}\right]=-i \hbar \hat{x}_{R}^{3}, \quad\left[\hat{x}_{R}^{5}, \hat{p}_{2}\right]=-\left[\hat{x}_{R}^{6}, \hat{p}_{1}\right]=i \hbar \hat{x}_{R}^{4}$.

For the compact case, the frame leads to the classical relations

$$
\begin{aligned}
& x^{3} \rightarrow x^{3}+2 \pi R^{3} \quad \Rightarrow \quad x^{5} \rightarrow x^{5}-2 \pi R^{3} x^{1}, \quad x^{6} \rightarrow x^{6}-2 \pi R^{3} x^{2}, \\
& x^{4} \rightarrow x^{4}+2 \pi R^{4} \quad \Rightarrow \quad x^{5} \rightarrow x^{5}+2 \pi R^{4} x^{2}, \quad x^{6} \rightarrow x^{6}-2 \pi R^{4} x^{1},
\end{aligned}
$$

which imply the following off-diagonal $\tau_{i}^{a}$ components for the noncommutative case:

$$
\tau_{3}^{5}=\tau_{4}^{6}=-\hat{x}_{R}^{1}, \quad \tau^{5}=-\tau_{3}^{6}=\hat{x}_{R}^{2} .
$$

The algebra in the compact case is easily constructed using Eqs. (3.37) and (3.38) and we do not write it explicitly. 
Step 5: $(\mathbf{0}, \mathbf{0}, \mathbf{1 2}, \mathbf{1 3}, \mathbf{1 4}+\mathbf{2 3}, \mathbf{1 5}+\mathbf{2 4})$. The last case we highlight is a step 5 nilmanifold (the last entry of Table 10 in the Appendix), which is the most complicated one and also the only one that leads to an $\hat{x}_{R}^{a}$-dependent quadratic term in the momentum commutator. We would like in particular to examine how the Jacobi identities are satisfied.

The 1-forms in this case are taken to be

$$
\begin{aligned}
& e^{1}=\mathrm{d} x^{1}, \quad e^{2}=\mathrm{d} x^{2}, \quad e^{3}=\mathrm{d} x^{3}+x^{2} \mathrm{~d} x^{1}, \quad e^{4}=\mathrm{d} x^{4}-x^{1} \mathrm{~d} x^{3}, \\
& e^{5}=\mathrm{d} x^{5}+\left(x^{4}-x^{1} x^{3}\right) \mathrm{d} x^{1}+\left(x^{3}+x^{1} x^{2}\right) \mathrm{d} x^{2}, \\
& e^{6}=\mathrm{d} x^{6}+\left(x^{5}+2 x^{2} x^{3}+\frac{1}{2} x^{1}\left(x^{2}\right)^{2}\right) \mathrm{d} x^{1}-\left(x^{4}-x^{1} x^{3}\right) \mathrm{d} x^{2}+2 x^{1} x^{2} \mathrm{~d} x^{3}-2 x^{2} \mathrm{~d} x^{4} .
\end{aligned}
$$

A direct computation shows that

$$
\omega=e^{16}+2 e^{34}-e^{25}=\mathrm{d} x^{16}+2 \mathrm{~d} x^{34}-\mathrm{d} x^{25}
$$

for the symplectic 2-form. Simlarly, the symplectic 2-vector satisfies

$$
\theta=\theta_{16}+\frac{1}{2} \theta_{34}-\theta_{25}=\partial_{16}+\frac{1}{2} \partial_{34}-\partial_{25},
$$

and the position commutators are

$$
\left[\hat{x}^{1}, \hat{x}^{6}\right]=\left[\hat{x}^{5}, \hat{x}^{2}\right]=i, \quad\left[\hat{x}^{3}, \hat{x}^{4}\right]=\frac{i}{2} .
$$

Finding the inverse of the frame,

$$
\begin{aligned}
& e_{i}^{a}=\left(\begin{array}{cccccc}
1 & 0 & 0 & 0 & 0 & 0 \\
0 & 1 & 0 & 0 & 0 & 0 \\
x^{2} & 0 & 1 & 0 & 0 & 0 \\
0 & 0 & -x^{1} & 1 & 0 & 0 \\
x^{4}-x^{1} x^{3} & x^{3}+x^{1} x^{2} & 0 & 0 & 1 & 0 \\
x^{5}+2 x^{2} x^{3}+\frac{1}{2} x^{1}\left(x^{2}\right)^{2} & -x^{4}+x^{1} x^{3} & 2 x^{1} x^{2} & -2 x^{2} & 0 & 1
\end{array}\right)^{-1} \\
& =\left(\begin{array}{cccccc}
1 & 0 & 0 & 0 & 0 & 0 \\
0 & 1 & 0 & 0 & 0 & 0 \\
-x^{2} & 0 & 1 & 0 & 0 & 0 \\
-x^{1} x^{2} & 0 & x^{1} & 1 & 0 & 0 \\
-x^{4}+x^{1} x^{3} & -x^{3}-x^{1} x^{2} & 0 & 0 & 1 & 0 \\
-x^{5}-2 x^{2} x^{3}-\frac{1}{2} x^{1}\left(x^{2}\right)^{2} & x^{4}-x^{1} x^{3} & 0 & 2 x^{2} & 0 & 1
\end{array}\right),
\end{aligned}
$$

provides the mixed commutators $\left[\hat{x}^{a}, \hat{p}_{i}\right]=i \hbar e_{i}^{a}\left(\hat{x}_{R}^{b}\right)$, which we do not write explicitly. The momenta are found to be

$$
\begin{aligned}
\hat{p}_{1}= & \hbar\left(\left[\hat{x}^{6}, \cdot\right]-2 \hat{x}_{R}^{2}\left[\hat{x}^{4}, \cdot\right]+2 \hat{x}_{R}^{1} \hat{x}_{R}^{2}\left[\hat{x}^{3}, \cdot\right]-\right. \\
& \left.\quad-\left(\hat{x}_{R}^{4}-\hat{x}_{R}^{1} \hat{x}_{R}^{3}\right)\left[\hat{x}^{2}, \cdot\right]+\left(\hat{x}_{R}^{5}+2 \hat{x}_{R}^{2} \hat{x}_{R}^{3}+\frac{1}{2} \hat{x}_{R}^{1}\left(\hat{x}_{R}^{2}\right)^{2}\right)\left[\hat{x}^{1}, \cdot\right]\right), \\
\hat{p}_{2}= & -\hbar\left(\left[\hat{x}^{5}, \cdot\right]+\left(\hat{x}_{R}^{3}+\hat{x}_{R}^{1} \hat{x}_{R}^{2}\right)\left[\hat{x}^{2}, \cdot\right]+\left(\hat{x}_{R}^{4}-\hat{x}_{R}^{1} \hat{x}_{R}^{3}\right)\left[\hat{x}^{1}, \cdot\right]\right) \\
\hat{p}_{3}= & 2 \hbar\left(\left[\hat{x}^{4}, \cdot\right]-\hat{x}_{R}^{1}\left[\hat{x}^{3}, \cdot\right]\right) \\
\hat{p}_{4}= & -2 \hbar\left(\left[\hat{x}^{3}, \cdot\right]+\hat{x}_{R}^{2}\left[\hat{x}^{1}, \cdot\right]\right) \\
\hat{p}_{5}= & \hbar\left[\hat{x}^{2}, \cdot\right], \quad \hat{p}_{6}=-\hbar\left[\hat{x}^{1}, \cdot\right]
\end{aligned}
$$


with quadratic commutation relations (we do not explicitly write the ones that are simply linear):

$$
\begin{aligned}
& {\left[\hat{p}_{1}, \hat{p}_{2}\right]=-i \hbar \hat{p}_{3}+\frac{i}{2}\left(\hat{p}_{5}\right)^{2}+i \hat{x}_{R}^{2}\left(\hat{p}_{6}\right)^{2}-i \hat{x}_{R}^{1} \hat{p}_{5} \hat{p}_{6}} \\
& {\left[\hat{p}_{1}, \hat{p}_{4}\right]=-i \hbar \hat{p}_{5}+2 i\left(\hat{p}_{6}\right)^{2}}
\end{aligned}
$$

We observe that the commutator (5.31) contains $\hat{x}_{R}^{a}$-dependent quadratic terms. In the present case, the Jacobi identities involve highly nontrivial cancellations. For example, $\operatorname{Jac}\left(\hat{p}_{1}, \hat{p}_{2}, \hat{x}_{R}^{5}\right)$ contains the terms

$$
\begin{aligned}
& {\left[\left[\hat{p}_{1}, \hat{p}_{2}\right], \hat{x}_{R}^{5}\right]=\hbar \hat{p}_{5}-\left(\hat{p}_{6}\right)^{2}-\hbar \hat{x}_{R}^{1} \hat{p}_{6},} \\
& {\left[\left[\hat{x}_{R}^{5}, \hat{p}_{1}\right], \hat{p}_{2}\right]=-\frac{3}{2} \hbar \hat{p}_{5}+\left(\hat{p}_{6}\right)^{2}-\hbar \hat{x}_{R}^{1} \hat{p}_{6},} \\
& {\left[\left[\hat{p}_{2}, \hat{x}_{R}^{5}\right], \hat{p}_{1}\right]=\frac{1}{2} \hbar \hat{p}_{5}+2 \hbar \hat{x}_{R}^{1} \hat{p}_{6},}
\end{aligned}
$$

and we observe that they sum to zero. A number of such cancellations occurs for the rest of the Jacobi identities too.

The treatment of the compact case follows the same lines as in the previous examples and therefore we do not present it explicitly.

\section{Discussion}

The main arena of quantum mechanics is phase space, which is quantized and without points in the classical sense of geometry, due to the uncertainty principle. On the other hand, general relativity accounts for the gravitational interaction by describing the dynamics of spacetime. The question of how these two theories become compatible is the most challenging conceptual problem in theoretical physics today. It is conceivable that one way that might illustrate the path towards quantum gravitational physics is to determine a framework where phase space and dynamical spacetime are reconciled in a dynamical theory of phase space (see e.g. Ref. [40] for a recent argumentation).

In this paper we employed an algebraic point of view and examined the algebraic properties of noncommutative phase spaces in the presence of a nontrivial frame. Although we did not study any dynamics, our results indicate that this can be possible at a later stage. In particular we showed that there exist consistent algebraic structures that incorporate quantized phase spaces with curvature and we studied a particular class of explicit examples. The new element that did not appear in previous similar approaches is that two copies of the noncommutative algebra are necessary, consisting of operators with a left and right action respectively. Most importantly, in curved cases these operators play an asymmetric role and satisfy different commutation relations with the momenta, a fact that is hidden in the flat case. This result should be taken into account in any attempt to construct a dynamical theory of phase space.

Considering that we are working in the framework of noncommutative geometry, one of the most motivating ways to think of an incarnation of such a dynamical theory of phase space is matrix models. Recall that in the well-known IKKT model [41], spacetime emerges 
dynamically (see e.g. Ref. [42] for a recent review). Moreover, the model can be quantized via an integral over matrices, given by the partition function

$$
\mathcal{Z}=\int \mathrm{d} A \mathrm{~d} \Psi e^{-S}, \quad S=-\frac{1}{4} \operatorname{Tr}\left[A_{M}, A_{N}\right]^{2}+S_{\text {matter }}
$$

with $A_{M}$ being ten Hermitian matrices. Correlation functions may be similarly defined and they are in principle computable with analytical or numerical methods. Classical solutions of the model are typically noncommutative spaces, where coordinate operators are identified with the matrices. On the other hand, according to the general arguments that we presented here, it would be preferable to obtain classical solutions that correspond to noncommutative phase spaces, such as the ones studied in this paper, in order to understand the role of the gravitational field too. Presumably, these are solutions of an extended matrix model that can account for the dynamics of phase space and can be quantized in a way similar to the above. We hope to report on this in a future publication.

In this paper we started with four basic assumptions, namely

- parallelizability, or equivalently existence of a globally well defined frame,

- symplectic structure,

- Leibniz rule and

- Jacobi identities.

Then we set the position commutators equal to the components of the symplectic 2-vector and implementing the frame by setting the mixed commutator between positions and momenta proportional to it. Consistency of these relations allowed us to determine the general form of the momentum operators as well as their commutation relation, which turned out to be quadratic in the momenta in accord with previous results [1].

In the process of our investigation we emphasized the distinct role of left and right acting operators and discussed the symplectic duality among the two sets. Although in simple cases, like the d-plane or the d-torus, this does not have any nontrivial consequences, departure from flatness breaks the symmetric role among the two. In particular, consistency of the formalism led us to associate the noncommutative frame to the set of right acting operators, when the observables of the theory lie in the pool of left acting ones. This made it necessary to consider an extended algebra of position operators $\hat{x}_{L}^{a}$, momentum operators $\hat{p}_{i}$ and quantized coordinate operators $\hat{x}_{R}^{a}$. This extended algebra was fully determined and it turned out that all the Jacobi identities are satisfied.

The general approach finds an elegant realization in a class of spaces which are known as nilmanifolds. These are iterated nontrivial fibrations of tori over tori and they yield several cases of symplectic manifolds in four and six dimensions. These symplectic cases were classified already in Ref. [37] and here we reclassified them according to their nilpotency step. Then we applied the general results and discussed some benchmark cases in detail.

In analogy to the compactification of a d-plane to a d-torus, manifolds based on nilpotent Lie algebras can be also compactified with a similar procedure based on identification 
conditions. It is well known that in compact cases, the position operators have to be exponentiated in order to be single valued. This holds true in the case of nilmanifolds too, although one has to be cautious about some additional complications due to the liberation between left and right operators. In particular it turned out that the identification conditions have to be imposed on the right operators, thus compactifying the noncommutative manifold, while the left acting operators are simply exponentiated, thus rendering the positions single valued. Similarly to the flat case, where two dual tori appear in the compactification process, in the curved case we encounter two dual nilmanifolds.

We already emphasized that the main goal would be to derive some dynamical theory of phase space that would be relevant for quantum gravity. Apart from this, there are four more immediate and clear paths that call for further investigation. First, an important next step of the present analysis is to define and compute the curvature of the quantized spaces in question. One can expect to derive an expression that converges to the classical curvature in the commutative limit but it carries more terms in the quantum case. A similar approach was employed in Refs. [8-10]. Such a task will also assist in understanding the common features and the differences with other recent approaches, such as [44-49]. Second, a different direction would be to go beyond the symplectic case. In general, nilmanifolds are not always symplectic, while symplectic nilmanifolds are not always only symplectic. At least in six dimensions, all nilmanifolds admit generalized complex structures, as proven in Ref. [38. This fact was used in the analysis of Dirac structures on step 2 nilmanifolds in Ref. [43]. The task would be to study the quantization of these structures as well. The third direction is to consider the quantization in the presence of sources. Although this is known for planes and tori, it is less obvious how straightforward it will be to implement sources for the nilmanifolds in the quantum case. A final possibility would be to go beyond nilmanifolds and examine what other spaces could be handled with the techniques of the present work. For example an obvious challenge is to examine symplectic solvmanifolds, which are more complicated that nilmanifolds.

Acknowledgements. The author would like to thank M. Burić, F.F. Gautason, L. Jonke, O. Lechtenfeld, J. Madore, P. Schupp and R. Szabo for discussions related to the content of the paper.

\section{Appendix}

\section{A Additional data for symplectic nilmanifolds}

In this Appendix we collect all the necessary additional data for the construction of the noncommutative phase space of symplectic nilmanifolds. We recall that this is given as

$$
\begin{aligned}
{\left[\hat{x}^{a}, \hat{x}^{b}\right] } & =i \theta^{a b} \\
{\left[\hat{x}^{a}, \hat{p}_{i}\right] } & =i \hbar e^{a}{ }_{i}, \\
{\left[\hat{p}_{i}, \hat{p}_{j}\right] } & =M_{i j}+N_{i j}{ }^{k} \hat{p}_{k}+P_{i j}^{k l} \hat{p}_{k} \hat{p}_{l} .
\end{aligned}
$$


The parameters $\theta^{a b}$ can be read off from the Tables 1-5 in the main text. They are antisymmetric and their value is \pm 1 along the directions of the symplectic structure. The parameters $e_{i}^{a}$ can be read off from the Tables 6-10 that we present in this Appendix, in the column titled "Frame: $e^{i}-\delta^{i}{ }_{a} \mathrm{~d} x^{a}$ ". The frame is written as a d-tuple $\left(\delta e^{1}{ }_{a}(x) \mathrm{d} x^{a}, \delta e_{a}^{2}(x) \mathrm{d} x^{a}, \ldots, \delta e_{a}^{\mathrm{d}}(x) \mathrm{d} x^{a}\right)$, where $\delta e^{i}{ }_{a}=e^{i}{ }_{a}-\delta^{i}{ }_{a}$. The parameters $M_{i j}$ are always vanishing, so they are not presented. Moreover, we have checked that the parameters that determine the linear term in the momentum commutator are generically given as

$$
N_{i j}^{k}=-i \hbar f_{i j}^{k} \text {. }
$$

This was proven analytically for step 2 nilmanifolds in Section 4.2, but it turns out that it holds for any step. Therefore it is unnecessary to present these parameters in the tables. Finally, we present the quadratic parameters $P_{i j}^{k l}$ and we remind that they are antisymmetric in the lower indices and symmetric in the upper ones. Note that the entries that do not satisfy the proposition of Section 4.2 are not examined and they are marked as " $N / A "$.

$\begin{array}{cccc}\text { Class } & \text { Step } & \frac{\text { Frame: } e^{i}-\delta^{i}{ }_{a} \mathrm{~d} x^{a}}{\left(0,0,0, x^{2} \mathrm{~d} x^{1}\right)} & \frac{P_{i j}^{k l}}{0} \\ (0,0,0,12) & 2 & \left.0,0,-\left(x^{4}+x^{1} x^{2}\right) \mathrm{d} x^{2}, x^{2} \mathrm{~d} x^{1}\right) & 0 \\ (0,0,42,12) & 3 & (0,0)\end{array}$

Table 6: Data for symplectic nilmanifolds in 4D.

\begin{tabular}{|ccc|}
\hline$\underline{\text { Class }}$ & $\frac{\text { Frame: } e^{i}-\delta^{i}{ }_{a} \mathrm{~d} x^{a}}{\left(0,0,0,0,0, x^{2} \mathrm{~d} x^{1}\right)}$ & $\frac{P_{i j}^{k l}}{0}$ \\
$(0,0,0,0,0,12)$ & $\left(0,0,0,0,-x^{1} \mathrm{~d} x^{2}, x^{3} \mathrm{~d} x^{1}\right)$ & 0 \\
$(0,0,0,0,13+42,14+23)$ & $\left(0,0,0,0, x^{3} \mathrm{~d} x^{1}-x^{4} \mathrm{~d} x^{2}, x^{4} \mathrm{~d} x^{1}+x^{3} \mathrm{~d} x^{2}\right)$ & $P_{12}^{55}=P_{12}^{66}=i$ \\
$(0,0,0,0,12,13)$ & $\left(0,0,0,0, x^{2} \mathrm{~d} x^{1}, x^{4} \mathrm{~d} x^{3}\right)$ & 0 \\
$(0,0,0,0,12,34)$ & $\left(0,0,0,0,-x^{1} \mathrm{~d} x^{2}, x^{3} \mathrm{~d} x^{2}-x^{1} \mathrm{~d} x^{4}\right)$ & $P_{13}^{56}=-i / 2$ \\
$(0,0,0,0,12,14+23)$ & $P_{24}^{66}=-i$ \\
$(0,0,0,12,13,23)$ & $\left(0,0,0,-x^{1} \mathrm{~d} x^{2}, x^{3} \mathrm{~d} x^{1},-x^{2} \mathrm{~d} x^{3}\right)$ & 0 \\
\hline
\end{tabular}

Table 7: Data for step 2 symplectic nilmanifolds in 6D. 


\begin{tabular}{|ccc|}
\hline Class & $\underline{\text { Frame: } e^{i}-\delta^{i}{ }_{a} \mathrm{~d} x^{a}}$ & $\frac{P_{i j}^{k l}}{0}$ \\
$(0,0,0,0,12,14+25)$ & $\left(0,0,0,0,-x^{1} \mathrm{~d} x^{2}, x^{5} \mathrm{~d} x^{2}-x^{1} \mathrm{~d} x^{4}\right)$ & 0 \\
$(0,0,0,0,12,15)$ & $\left(0,0,0,0,-x^{1} \mathrm{~d} x^{2},\left(x^{5}-x^{1} x^{2}\right) \mathrm{d} x^{1}\right)$ & 0 \\
$(0,0,0,12,14+23,13+42)$ & $\left(0,0,0,-x^{1} \mathrm{~d} x^{2},\left(x^{4}-x^{1} x^{2}\right) \mathrm{d} x^{1}+x^{3} \mathrm{~d} x^{2}\right.$, & $P_{12}^{66}=\frac{i}{2}$ \\
$(0,0,0,12,14,13+42)$ & $\left(0,0,0,-x^{1} \mathrm{~d} x^{2},\left(x^{4}-x^{1} x^{2}\right) \mathrm{d} x^{1},-x^{4} \mathrm{~d} x^{2}-x^{1} \mathrm{~d} x^{3}\right)$ & 0 \\
$(0,0,0,12,14,23+24)$ & $\left(0,0,0, x^{2} \mathrm{~d} x^{1}, x^{4} \mathrm{~d} x^{1},-\frac{1}{2}\left(x^{2}\right)^{2} \mathrm{~d} x^{1}+x^{4} \mathrm{~d} x^{2}-x^{2} \mathrm{~d} x^{3}\right)$ & 0 \\
$(0,0,0,12,13,14)$ & $\left(0,0,0,-x^{1} \mathrm{~d} x^{2},-x^{1} \mathrm{~d} x^{3},\left(x^{4}-x^{1} x^{2}\right) \mathrm{d} x^{1}\right)$ & 0 \\
$(0,0,0,12,13,24)$ & $\left(0,0,0, x^{2} \mathrm{~d} x^{1},-x^{1} \mathrm{~d} x^{3},\left(x^{4}+x^{1} x^{2}\right) \mathrm{d} x^{2}\right)$ & $P_{23}^{56}=-\frac{i}{2}$ \\
$(0,0,0,12,13,14+23)$ & $\left(0,0,0, \frac{1}{2} x^{2} \mathrm{~d} x^{1}-\frac{1}{2} x^{1} \mathrm{~d} x^{2},-x^{1} \mathrm{~d} x^{3}\right.$, & 0 \\
\hline
\end{tabular}

Table 8: Data for step 3 symplectic nilmanifolds in 6D.

\begin{tabular}{|ccc|}
\hline Class & Frame: $e^{i}-\delta^{i}{ }_{a} \mathrm{~d} x^{a}$ & $\underline{P_{i j}^{k l}}$ \\
$(0,0,0,12,14-23,15+34)$ & $\left(0,0,0,-x^{1} \mathrm{~d} x^{2},\left(x^{4}-x^{1} x^{2}\right) \mathrm{d} x^{1}+x^{2} \mathrm{~d} x^{3}\right.$, & $P_{13}^{55}=i$ \\
$(0,0,0,12,14,15)$ & $\left.x^{5} \mathrm{~d} x^{1}+\left(x^{4}-x^{1} x^{2}\right) \mathrm{d} x^{3}\right)$ & \\
& $\left(0,0,0,-x^{1} \mathrm{~d} x^{2}, \frac{1}{2}\left(x^{1}\right)^{2} \mathrm{~d} x^{2}-x^{1} \mathrm{~d} x^{4}\right.$, & \\
$(0,0,0,12,14,15+24)$ & $\left.-x^{1} \mathrm{~d} x^{5}+\frac{1}{2}\left(x^{1}\right)^{2} \mathrm{~d} x^{4}-\frac{1}{6}\left(x^{1}\right)^{3} \mathrm{~d} x^{2}\right)$ & 0 \\
& $\left(0,0,0,-x^{1} \mathrm{~d} x^{2}, \frac{1}{2}\left(x^{1}\right)^{2} \mathrm{~d} x^{2}-x^{1} \mathrm{~d} x^{4}\right.$, & \\
$(0,0,0,12,14,15+23+24)$ & $\left.x^{4} \mathrm{~d} x^{2}-x^{1} \mathrm{~d} x^{5}+\frac{1}{2}\left(x^{1}\right)^{2} \mathrm{~d} x^{4}-\frac{1}{6}\left(x^{1}\right)^{3} \mathrm{~d} x^{2}\right)$ & $P_{24}^{56}=-\frac{i}{2}$ \\
$(0,0,0,12,14,23+15)$ & $\left(0,0,0,-x^{1} \mathrm{~d} x^{2}, \frac{1}{2}\left(x^{1}\right)^{2} \mathrm{~d} x^{2}-x^{1} \mathrm{~d} x^{4}\right.$, & $P_{25}^{66}=-i$ \\
$(0,0,12,13,23,14)$ & $\left(0,0,0,-x^{1} \mathrm{~d} x^{2}, \frac{1}{2}\left(x^{1}\right)^{2} \mathrm{~d} x^{2}-x^{1} \mathrm{~d} x^{4}\right.$, & $P_{24}^{56}=-\frac{i}{2}$ \\
$(0,0,12,13,23,14-25)$ & $\left.\left.x^{3}+x^{4}\right) \mathrm{d} x^{2}-x^{1} \mathrm{~d} x^{5}+\frac{1}{2}\left(x^{1}\right)^{2} \mathrm{~d} x^{4}-\frac{1}{6}\left(x^{1}\right)^{3} \mathrm{~d} x^{2}\right)$ & $P_{25}^{66}=-i$ \\
$(0,0,12,13,23,14+25)$ & $N / A$ & \\
\hline
\end{tabular}

Table 9: Data for step 4 symplectic nilmanifolds in 6D. 


\begin{tabular}{|ccc|}
\hline Class & $\frac{\text { Frame: } e^{i}-\delta^{i}{ }_{a} \mathrm{~d} x^{a}}{P_{i j}^{k l}}$ \\
& $\left(0,0,-x^{1} \mathrm{~d} x^{2},-x^{1} \mathrm{~d} x^{3}+\frac{1}{2}\left(x^{1}\right)^{2} \mathrm{~d} x^{2}\right.$, & \\
$(0,0,12,13,14,15)$ & $-x^{1} \mathrm{~d} x^{4}+\frac{1}{2}\left(x^{1}\right)^{2} \mathrm{~d} x^{3}-\frac{1}{6}\left(x^{1}\right)^{3} \mathrm{~d} x^{2}$, & 0 \\
$(0,0,12,13,14,15+23)$ & $\left.\left(x^{5}-x^{1} x^{4}+\frac{1}{2}\left(x^{1}\right)^{2} x^{3}-\frac{1}{6}\left(x^{1}\right)^{3} x^{2}\right) \mathrm{d} x^{1}\right)$ & \\
$N / A$ & \\
$(0,0,12,13,14+23,15+24)$ & $\left(0,0, x^{2} \mathrm{~d} x^{1},-x^{1} \mathrm{~d} x^{3}\right.$, & \\
& $\left(x^{4}-x^{1} x^{3}\right) \mathrm{d} x^{1}+\left(x^{3}+x^{1} x^{2}\right) \mathrm{d} x^{2}$, & $P_{12}^{55}=\frac{i}{2}$ \\
& $\left(x^{5}+2 x^{2} x^{3}+\frac{1}{2} x^{1}\left(x^{2}\right)^{2}\right) \mathrm{d} x^{1}$ & $P_{14}^{66}=2 i$ \\
$-\left(x^{4}-x^{1} x^{3}\right) \mathrm{d} x^{2}$ & $P_{12}^{66}=i \hat{x}_{R}^{2}$ \\
& $\left.+2 x^{1} x^{2} \mathrm{~d} x^{3}-2 x^{2} \mathrm{~d} x^{4}\right)$ & $P_{12}^{56}=-\frac{i}{2} \hat{x}_{R}^{1}$ \\
\hline
\end{tabular}

Table 10: Data for step 5 symplectic nilmanifolds in 6D.

\section{References}

[1] J. Madore, An introduction to noncommutative differential geometry and its physical applications, Lond. Math. Soc. Lect. Note Ser. 257 (2000) 1.

[2] A. Connes, Noncommutative geometry, Academic Press, San Diego, CA, 1994, 661p.

[3] R. J. Szabo, Phys. Rept. 378 (2003) 207 hep-th/0109162].

[4] A. Connes, M. R. Douglas and A. S. Schwarz, JHEP 9802 (1998) 003 hep-th/9711162.

[5] N. Seiberg and E. Witten, JHEP 9909 (1999) 032 [hep-th/9908142].

[6] R. Blumenhagen, arXiv:1403.4805 [hep-th].

[7] S. Doplicher, K. Fredenhagen and J. E. Roberts, Commun. Math. Phys. 172 (1995) 187 [hep-th/0303037].

[8] M. Burić and J. Madore, Gen. Rel. Grav. 43 (2011) 3477 arXiv:1110.0592 [hep-th]].

[9] M. Burić, T. Grammatikopoulos, J. Madore and G. Zoupanos, JHEP 0604 (2006) 054 hep-th/0603044.

[10] M. Burić, J. Madore and G. Zoupanos, Eur. Phys. J. C 55 (2008) 489 arXiv:0709.3159 [hep-th]].

[11] C. Duval and P. A. Horvathy, Phys. Lett. B 479 (2000) 284 hep-th/0002233.

[12] V. P. Nair and A. P. Polychronakos, Phys. Lett. B 505 (2001) 267 hep-th/0011172.

[13] B. Morariu and A. P. Polychronakos, Nucl. Phys. B 610 (2001) 531 hep-th/0102157.

[14] P. A. Horvathy, Annals Phys. 299 (2002) 128 hep-th/0201007. 
[15] P. A. Horvathy, L. Martina and P. C. Stichel, SIGMA 6 (2010) 060 arXiv:1002.4772 [hep-th]].

[16] D. Karabali, V. P. Nair and A. P. Polychronakos, Nucl. Phys. B 627 (2002) 565 hep-th/0111249].

[17] S. -C. Zhang and J. -p. Hu, Science 294 (2001) 823 [cond-mat/0110572].

[18] D. Karabali and V. P. Nair, Nucl. Phys. B 641 (2002) 533 hep-th/0203264.

[19] A. Chou, C. Hogan, E. Ramberg, J. Steffen, C. Stoughton, R. Tomlin, W. Wester and S. Waldman et al., "The Fermilab Holometer," FERMILAB-PROPOSAL-0990.

[20] I. Ruo Berchera, I. P. Degiovanni, S. Olivares and M. Genovese, Phys. Rev. Lett. 110 213601 (2013) [arXiv:1304.7912 [quant-ph]].

[21] M. Kontsevich, Lett. Math. Phys. 66 (2003) 157 arXiv:q-alg/9709040 [q-alg]].

[22] J. Madore, S. Schraml, P. Schupp and J. Wess, Eur. Phys. J. C 16 (2000) 161 hep-th/0001203.

[23] S. Bates and A. Weinstein, Lectures on the Geometry of Quantization, Berkeley Mathematics Lecture Notes series Vol. 8, American Mathematical Society 1997.

[24] V. P. Nair, Phys. Lett. B 505 (2001) 249 hep-th/0008027.

[25] D. J. Gross and N. A. Nekrasov, JHEP 0007 (2000) 034 hep-th/0005204].

[26] D. Brace, B. Morariu and B. Zumino, Nucl. Phys. B 545 (1999) 192 hep-th/9810099.

[27] P. Ramond, Field Theory. A Modern Primer, Front. Phys. 51 (1981) 1.

[28] A. Chatzistavrakidis and L. Jonke, Phys. Rev. D 85 (2012) 106013 arXiv:1202.4310 [hep-th]].

[29] A. Chatzistavrakidis and L. Jonke, JHEP 1302 (2013) 040 [arXiv:1207.6412 [hep-th]].

[30] J. Madore, Class. Quant. Grav. 9 (1992) 69.

[31] S. Ramgoolam, Nucl. Phys. B 610 (2001) 461 [hep-th/0105006].

[32] S. Ramgoolam, JHEP 0210 (2002) 064 hep-th/0207111.

[33] K. Hasebe, SIGMA 6 (2010) 071 [arXiv:1009.1192 [hep-th]].

[34] M. A. Rieffel, Comm. Math. Phys. Volume 122, Number 4 (1989), 531-562.

[35] D. A. Lowe, H. Nastase and S. Ramgoolam, Nucl. Phys. B 667 (2003) 55 hep-th/0303173.

[36] J. Patera, R. T. Sharp, P. Winternitz, H. Zassenhaus, J. Math. Phys. 17 (1976) 986. 
[37] M. Goze, Y. Khakimdjanov, Nilpotent Lie Algebras, Mathematics and its Applications, 361, Kluwer Academic Publishers Group, Dordrecht, 1996.

[38] G.R. Cavalcanti and M. Gualtieri, J. Symplectic Geom. 2 (2004) 393 arXiv:math/0404451 [math.DG]].

[39] C. Benson, C. S. Gordon, Topology, Volume 27, Issue 4, 1988, Pages 513-518.

[40] L. Freidel, R. G. Leigh and D. Minic, "Quantum Gravity, Dynamical Phase Space and String Theory," arXiv:1405.3949 [hep-th].

[41] N. Ishibashi, H. Kawai, Y. Kitazawa and A. Tsuchiya, Nucl. Phys. B 498 (1997) 467 hep-th/9612115.

[42] J. Nishimura, PTEP 2012 (2012) 01A101 [arXiv:1205.6870 [hep-lat]].

[43] A. Chatzistavrakidis, L. Jonke and O. Lechtenfeld, Nucl. Phys. B 883 (2014) 59 arXiv:1311.4878 [hep-th]].

[44] H. Steinacker, Class. Quant. Grav. 27 (2010) 133001 [arXiv:1003.4134 [hep-th]].

[45] D. N. Blaschke and H. Steinacker, Class. Quant. Grav. 27 (2010) 165010 arXiv:1003.4132 [hep-th]].

[46] D. N. Blaschke and H. Steinacker, Class. Quant. Grav. 27 (2010) 235019 arXiv:1007.2729 [hep-th]].

[47] P. Aschieri and L. Castellani, JHEP 0906 (2009) 086 [arXiv:0902.3817 [hep-th]].

[48] P. Aschieri and L. Castellani, Gen. Rel. Grav. 45 (2013) 411 [arXiv:1206.4096 [hep-th]].

[49] H. S. Yang, arXiv:1312.0580 [hep-th]. 